\title{
Energy asymptotics in the three-dimensional Brezis-Nirenberg problem
}

\author{
Rupert L. Frank ${ }^{1,2}$ (D) $\cdot$ Tobias König ${ }^{3} \cdot$ Hynek Kovařík ${ }^{4}$
}

Received: 9 October 2020 / Accepted: 25 January 2021 / Published online: 19 February 2021

(c) The Author(s) 2021

\section{Abstract}

For a bounded open set $\Omega \subset \mathbb{R}^{3}$ we consider the minimization problem

$$
S(a+\epsilon V)=\inf _{0 \neq u \in H_{0}^{1}(\Omega)} \frac{\int_{\Omega}\left(|\nabla u|^{2}+(a+\epsilon V)|u|^{2}\right) d x}{\left(\int_{\Omega} u^{6} d x\right)^{1 / 3}}
$$

involving the critical Sobolev exponent. The function $a$ is assumed to be critical in the sense of Hebey and Vaugon. Under certain assumptions on $a$ and $V$ we compute the asymptotics of $S(a+\epsilon V)-S$ as $\epsilon \rightarrow 0+$, where $S$ is the Sobolev constant. (Almost) minimizers concentrate at a point in the zero set of the Robin function corresponding to $a$ and we determine the location of the concentration point within that set. We also show that our assumptions are almost necessary to have $S(a+\epsilon V)<S$ for all sufficiently small $\epsilon>0$.

Mathematics Subject Classification 35A15 $\cdot 35 \mathrm{~J} 20 \cdot 35 \mathrm{~B} 44$

Communicated by Andrea Malchiodi.

(C) 2020 by the authors. This paper may be reproduced, in its entirety, for non-commercial purposes. Partial support through US National Science Foundation Grants DMS-1363432 and DMS-1954995 (R.L.F.),

Studienstiftung des deutschen Volkes (T.K.) and Gruppo Nazionale per Analisi Matematica, la Probabilità e le loro Applicazioni (GNAMPA) of the Istituto Nazionale di Alta Matematica (INdAM) (H.K.) is acknowledged.

$\bowtie$ Rupert L. Frank

r.frank@1mu.de; rlfrank@caltech.edu

Tobias König

koenig@imj-prg.fr

Hynek Kovařík

hynek.kovarik@unibs.it

1 Mathematisches Institut, Ludwig-Maximilians-Universität München, Theresienstr. 39, 80333

Munich, Germany

2 Mathematics 253-37, Caltech, Pasadena, CA 91125, USA

3 Institut de Mathématiques de Jussieu - Paris Rive Gauche, Université de Paris, Bâtiment Sophie Germain, Case 7012, 75205 Paris Cedex 13, France

4 DICATAM, Sezione di Matematica, Università degli studi di Brescia, Brescia, Italy 


\section{Introduction and main results}

\subsection{Setting of the problem}

In their celebrated paper [8] Brézis and Nirenberg considered the problem of minimizing the quotient

$$
\mathcal{S}_{a}[u]:=\frac{\int_{\Omega}\left(|\nabla u|^{2}+a|u|^{2}\right) d x}{\left(\int_{\Omega} u^{6} d x\right)^{1 / 3}}
$$

over all $0 \not \equiv u \in H_{0}^{1}(\Omega)$, where $\Omega \subset \mathbb{R}^{3}$ is a bounded open set and $a$ is a continuous function on $\bar{\Omega}$. We denote the corresponding infimum by

$$
S(a):=\inf _{0 \neq u \in H_{0}^{1}(\Omega)} \mathcal{S}_{a}[u] .
$$

This number is to be compared with

$$
S:=3\left(\frac{\pi}{2}\right)^{4 / 3},
$$

the sharp constant $[3,25,26,31]$ in the Sobolev inequality

$$
\int_{\mathbb{R}^{3}}|\nabla u|^{2} d x \geq S\left(\int_{\mathbb{R}^{3}} u^{6} d x\right)^{1 / 3}, \quad u \in \dot{H}^{1}\left(\mathbb{R}^{3}\right) .
$$

One of the findings in [8] is that if $a$ is small (for instance, in $L^{\infty}(\Omega)$ ), then $S(a)=S$. This is in stark contrast to the case of dimensions $N \geq 4$ where the corresponding analogue of $S(a)$ (with the exponent 6 replaced by $2 N /(N-2)$ ) is always strictly below the corresponding Sobolev constant, whenever $a$ is negative somewhere.

This phenomenon leads naturally to the following notion due to Hebey and Vaugon [20].

Definition 1.1 Let $a$ be a continuous function on $\bar{\Omega}$. We say that $a$ is critical in $\Omega$ if $S(a)=S$ and if for any continuous function $\tilde{a}$ on $\bar{\Omega}$ with $\tilde{a} \leq a$ and $\tilde{a} \not \equiv a$ one has $S(\tilde{a})<S(a)$.

Our goal in this paper is to compute the asymptotics of $S(a+\epsilon V)-S$ as $\epsilon \rightarrow 0$ for critical $a$ and to understand the behavior of corresponding minimizers. Here $V$ is a bounded function on $\Omega$, without any restrictions on its sign.

A key role in our analysis is played by the regular part of the Green's function and its zero set. To introduce these, we follow the sign and normalization convention of [24]. If the operator $-\Delta+a$ in $\Omega$ with Dirichlet boundary conditions is coercive (which, in particular, is the case if $a$ is critical), then it has a Green's function $G_{a}$ satisfying

$$
\begin{cases}-\Delta_{x} G_{a}(x, y)+a(x) G_{a}(x, y)=4 \pi \delta_{y} & \text { in } \Omega, \\ G_{a}(x, y)=0 & \text { on } \partial \Omega .\end{cases}
$$

The regular part of $G_{a}$ is defined by

$$
H_{a}(x, y):=\frac{1}{|x-y|}-G_{a}(x, y) .
$$

It is well-known that for each $x \in \Omega$ the function $H_{a}(x, \cdot)$, which is originally defined in $\Omega \backslash\{x\}$, extends to a continuous function in $\Omega$ and we abbreviate

$$
\phi_{a}(x):=H_{a}(x, x) .
$$


It has been understood that the function $\phi_{a}$ is relevant for problems involving the critical Sobolev exponent, see, e.g., [27] and [4]. For the problem at hand, it was shown in [6, Thm. 7] that if $\phi_{a}(x)<0$ for some $x \in \Omega$, then $S(a)<S$. (In [6] this is attributed to Schoen [27] and a work in preparation by McLeod.) Conversely, it was conjectured in [6] and proved by Druet in [12] that if $S(a)<S$, then $\phi_{a}(x)<0$ for some $x \in \Omega$. An alternative proof, assuming only continuity of $a$, is given in [15]. Thus, the (non-local) condition $\min _{\Omega} \phi_{a}<0$ is necessary and sufficient for $S(a)<S$, and replaces the (local) condition $\min _{\Omega} a<0$ in dimensions $N \geq 4$.

The above results imply that, if $a$ is critical, then $\min _{\Omega} \phi_{a}=0$. In particular, the set

$$
\mathcal{N}_{a}:=\left\{x \in \Omega: \phi_{a}(x)=0\right\}
$$

is non-empty.

\subsection{Main results}

Let us proceed to a precise statement of our main results. Throughout this paper we work under the following assumption.

Assumption 1.2 The set $\Omega \subset \mathbb{R}^{3}$ is open, bounded and has a $C^{2}$ boundary. The function $a$ satisfies $a \in C(\bar{\Omega}) \cap C^{1}(\Omega)$ and is critical in $\Omega$. Moreover,

$$
a(x)<0 \text { for all } x \in \mathcal{N}_{a} .
$$

Finally, $V \in L^{\infty}(\Omega)$.

We will see in Corollary 2.2 that criticality of $a$ alone implies $a(x) \leq 0$ for all $x \in \mathcal{N}_{a}$. Therefore assumption (1.4) is not severe.

We set

$$
Q_{V}(x):=\int_{\Omega} V(y) G_{a}(x, y)^{2} d y, \quad x \in \Omega,
$$

and

$$
\mathcal{N}_{a}(V):=\left\{x \in \mathcal{N}_{a}: Q_{V}(x)<0\right\}
$$

The following is our main result.

Theorem 1.3 Assume that $\mathcal{N}_{a}(V) \neq \emptyset$. Then $S(a+\epsilon V)<S$ for all $\epsilon>0$ and

$$
\lim _{\epsilon \rightarrow 0+} \frac{S(a+\epsilon V)-S}{\epsilon^{2}}=-\left(\frac{3}{S}\right)^{\frac{1}{2}} \frac{1}{8 \pi^{2}} \sup _{x \in \mathcal{N}_{a}(V)} \frac{Q_{V}(x)^{2}}{|a(x)|} .
$$

We supplement this theorem with a result for the opposite case where $\mathcal{N}_{a}(V)=\emptyset$.

Theorem 1.4 Assume that $\mathcal{N}_{a}(V)=\emptyset$. Then $S(a+\epsilon V)=S+o\left(\epsilon^{2}\right)$ as $\epsilon \rightarrow 0+$. If, in addition, $Q_{V}(x)>0$ for all $x \in \mathcal{N}_{a}$, then $S(a+\epsilon V)=S$ for all sufficiently small $\epsilon>0$.

It follows from the above two theorems that the condition $\mathcal{N}_{a}(V) \neq \emptyset$ is 'almost' necessary for the inequality $S(a+\epsilon V)<S$ for all small $\epsilon>0$. Only the case where $\min _{\mathcal{N}_{a}} Q_{V}=0$ is left open. 
Example 1.5 When $\Omega=B$ is the unit ball in $\mathbb{R}^{3}$, then it is well-known that the constant function $a=-\pi^{2} / 4$ is critical and that in this case $\mathcal{N}_{a}=\{0\}$ and $G_{a}(0, y)=|y|^{-1} \cos (\pi|y| / 2)$; see, e.g., [6]. Thus, with

$$
q_{V}:=Q_{V}(0)=\int_{B} V(y) \frac{\cos ^{2}(\pi|y| / 2)}{|y|^{2}} d y
$$

we have

$$
\lim _{\epsilon \rightarrow 0+} \frac{S(a+\epsilon V)-S}{\epsilon^{2}}=-\left(\frac{3}{S}\right)^{\frac{1}{2}} \frac{1}{2 \pi^{4}} q_{V}^{2} \text { if } q_{V} \leq 0
$$

and $S(a+\epsilon V)=S$ for all sufficiently small $\epsilon>0$ if $q_{V}>0$.

Remark 1.6 It is instructive to compare our results here with the results for the analogous problem

$$
S(\epsilon V):=\inf _{0 \neq \equiv \in H_{0}^{1}(\Omega)} \frac{\int_{\Omega}\left(|\nabla u|^{2}+\epsilon V u^{2}\right) d x}{\left(\int_{\Omega}|u|^{2 N /(N-2)} d x\right)^{(N-2) / N}}
$$

in dimension $N \geq 4$. Let $S_{N}$ be the sharp constant in the Sobolev inequality in $\mathbb{R}^{N}$. From [8] we know that $S(\epsilon V)<S_{N}$ if and only if $V(x)<0$ for some $x \in \Omega$, and therefore we focus on the case where $\mathcal{N}(V):=\{x \in \Omega: V(x)<0\} \neq \emptyset$. Then

$$
\begin{aligned}
& S(\epsilon V)=S_{N}-C_{N} \sup _{x \in \mathcal{N}(V)} \frac{|V(x)|^{\frac{N-2}{N-4}}}{\phi_{0}(x)^{\frac{2}{N-4}}} \epsilon^{\frac{N-2}{N-4}}+o\left(\epsilon^{\frac{N-2}{N-4}}\right) \quad \text { if } N \geq 5, \\
& S(\epsilon V)=S_{N}-\exp \left(-\frac{4}{\epsilon}(1+o(1)) \inf _{x \in \mathcal{N}(V)} \frac{\phi_{0}(x)}{|V(x)|}\right) \quad \text { if } N=4,
\end{aligned}
$$

with explicit constants $C_{N}$ depending only on $N$. Note that, as a reflection of the BrézisNirenberg phenomenon, $V$ enters pointwisely into the asymptotic coefficient in (1.7) and (1.8), while it enters non-locally through $Q_{V}$ into the asymptotic coefficient in Theorem 1.3.

Asymptotics (1.7) and (1.8) in the case where $V$ is a negative constant are essentially contained in [30]; see also [32] for related results. The case of general $V \in C(\bar{\Omega})$ can be treated by similar methods. For details, we refer to [18]. We emphasize that the proof of Theorem 1.3 is considerably more complicated than that of (1.7) and (1.8), since the expansion in Theorem 1.3 should rather be thought of as a higher order expansion of $S(a+\epsilon V)-S$ where the coefficient of the term of order $\epsilon$ vanishes due to criticality. In the higher dimensional context, no such cancellation occurs.

\subsection{Behavior of almost minimizers}

We prove Theorems 1.3 and 1.4 by proving upper and lower bounds on $S(a+\epsilon V)$. For the upper bound it suffices to evaluate $\mathcal{S}_{a+\epsilon V}\left[u_{\epsilon}\right]$ for an appropriately chosen family of functions $u_{\epsilon}$. For the lower bound we need to evaluate the same quantity where now $u_{\epsilon}$ is an optimizer for $S(a+\epsilon V)$. To do so, we will show that $u_{\epsilon}$ is essentially of the same form as the family chosen to prove the upper bound. In fact, we will not use the minimality of the $u_{\epsilon}$ and show that, more generally, all 'almost minimizers' have essentially the same form as the functions chosen for the upper bound. 
Given earlier works and, in particular, those by Druet [12] and Esposito [15] it is not surprising that almost minimizers concentrate at a point in the set $\mathcal{N}_{a}$. One of our new contributions is to show that this concentration happens at a point in the subset $\mathcal{N}_{a}(V)$ and, more precisely, at a point in $\mathcal{N}_{a}(V)$ where the supremum in (1.6) is attained.

In order to state our theorem about almost minimizers, for $x \in \Omega$ and $\lambda>0$, let

$$
U_{x, \lambda}(y):=\frac{\lambda^{1 / 2}}{\left(1+\lambda^{2}|y-x|^{2}\right)^{1 / 2}} .
$$

The functions $U_{x, \lambda}$ and their multiples are precisely the optimizers of the Sobolev inequality (1.1); see the references mentioned above and [22, Cor. I.1]. We introduce $P U_{x, \lambda} \in H_{0}^{1}(\Omega)$ as the unique function satisfying

$$
\Delta P U_{x, \lambda}=\Delta U_{x, \lambda} \quad \text { in } \Omega, \quad P U_{x, \lambda}=0 \quad \text { on } \partial \Omega .
$$

Moreover, let

$$
T_{x, \lambda}:=\operatorname{span}\left\{P U_{x, \lambda}, \partial_{\lambda} P U_{x, \lambda}, \partial_{x_{i}} P U_{x, \lambda}(i=1,2,3)\right\}
$$

and let $T_{x, \lambda}^{\perp}$ be the orthogonal complement of $T_{x, \lambda}$ in $H_{0}^{1}(\Omega)$ with respect to the inner product $\int_{\Omega} \nabla u \cdot \nabla v d y$. Finally, by $\Pi_{x, \lambda}$ and $\Pi_{x, \lambda}^{\perp}$ we denote the orthogonal projections in $H_{0}^{1}(\Omega)$ onto $T_{x, \lambda}$ and $T_{x, \lambda}^{\perp}$, respectively.

Theorem 1.7 Assume that $\mathcal{N}_{a}(V) \neq \emptyset$. Let $\left(u_{\epsilon}\right) \subset H_{0}^{1}(\Omega)$ be a family of functions such that

$$
\lim _{\epsilon \rightarrow 0} \frac{\mathcal{S}_{a+\epsilon V}\left[u_{\epsilon}\right]-S(a+\epsilon V)}{S-S(a+\epsilon V)}=0 \text { and } \int_{\Omega} u_{\epsilon}^{6} d x=\left(\frac{S}{3}\right)^{\frac{3}{2}} .
$$

Then there are $\left(x_{\epsilon}\right) \subset \Omega,\left(\lambda_{\epsilon}\right) \subset(0, \infty)$ and $\left(\alpha_{\epsilon}\right) \subset \mathbb{R}$ such that

$$
u_{\epsilon}=\alpha_{\epsilon}\left(P U_{x_{\epsilon}, \lambda_{\epsilon}}-\lambda_{\epsilon}^{-1 / 2} \Pi_{x_{\epsilon}, \lambda_{\epsilon}}^{\perp}\left(H_{a}\left(x_{\epsilon}, \cdot\right)-H_{0}\left(x_{\epsilon}, \cdot\right)\right)+r_{\epsilon}\right)
$$

and, along a subsequence,

$$
\begin{aligned}
x_{\epsilon} & \rightarrow x_{0} \text { for some } x_{0} \in \mathcal{N}_{a}(V) \text { with } \frac{Q_{V}\left(x_{0}\right)^{2}}{\left|a\left(x_{0}\right)\right|}=\sup _{y \in \mathcal{N}_{a}(V)} \frac{Q_{V}(y)^{2}}{|a(y)|}, \\
\phi_{a}\left(x_{\epsilon}\right) & =o(\epsilon), \\
\lim _{\epsilon \rightarrow 0} \epsilon \lambda_{\epsilon} & =4 \pi^{2} \frac{\left|a\left(x_{0}\right)\right|}{\left|Q_{V}\left(x_{0}\right)\right|} \\
\alpha_{\epsilon} & =s+\mathcal{O}(\epsilon) \text { for some } s \in\{ \pm 1\} .
\end{aligned}
$$

Finally, $r_{\epsilon} \in T_{x_{\epsilon}, \lambda_{\epsilon}}^{\perp}$ and $\left\|\nabla r_{\epsilon}\right\|=o(\epsilon)$.

The $L^{6}$ normalization in (1.10) is chosen in view of

$$
\int_{\mathbb{R}^{3}} U_{x, \lambda}^{6} d y=\left(\frac{S}{3}\right)^{\frac{3}{2}}
$$

There is a huge literature on blow-up results for solutions of equations involving the critical Sobolev exponent. Early contributions related to the problem we are considering are, for instance, $[2,9,10,19,23]$; see also the book [13] for more recent developments and further references. Here we follow a somewhat different philosophy and focus not on the equation satisfied by the minimizers, but solely on their minimality property. Therefore our proofs also 
apply to almost minimizers in the sense of (1.10) and we obtain blow-up results for those as well. This extension is not really necessary for the proof of our main results, Theorems 1.3 and 1.4, but it is crucial when studying parabolic or hyperbolic versions of the problem studied here. On the other hand, with our variational methods we cannot say anything about non-minimizing solutions of the corresponding equation and our blow-up bounds are only obtained in $H^{1}$ instead of $L^{\infty}$ norm. Other related works that study Sobolev critical problems from a variational point of view are, for instance, $[1,16,17]$.

As already mentioned before, the works of Druet [12] and Esposito [15], and similarly $[1,17]$ in related problems, show that concentration happens at a point in $\mathcal{N}_{a}$. In terms of $S(a+\epsilon V)$, this corresponds essentially to the fact that $S(a+\epsilon V)=S+o(\epsilon)$. In order to go further than that and to compute the coefficient of $\epsilon^{2}$, we need to prove that concentration happens in the subset $\mathcal{N}_{a}(V)$ at a point where the supremum in (1.6) is attained.

The strategy of the proof of the lower bound is to expand the quotient $\mathcal{S}_{a+\epsilon V}\left[u_{\epsilon}\right]$ for an almost minimizer $u_{\epsilon}$ as precisely as allowed by the available information on $u_{\epsilon}$, then to use a coercivity bound to deduce that certain terms are small and thereby improving our knowledge about $u_{\epsilon}$. We repeat this procedure three times (namely, in Sects. 4, 5 and 6). Therefore, a key tool in our analysis is the coercivity of the quadratic form

$$
\int_{\Omega}\left(|\nabla v|^{2}+a v^{2}-15 U_{x, \lambda}^{4} v^{2}\right) d x, \quad v \in T_{x, \lambda}^{\perp},
$$

provided that $\lambda \operatorname{dist}(x, \partial \Omega)$ is sufficiently large; see Lemma 4.3. This coercivity was proved by Esposito [15] and comes ultimately from the non-degeneracy of the Sobolev minimizer $U_{x, \lambda}$. Esposito used this bound to obtain an a priori bound on the term $\alpha_{\epsilon}^{-1} u_{\epsilon}-P U_{x_{\epsilon}, \lambda_{\epsilon}}$ in Theorem 1.7. We will use it for the same purpose in Proposition 4.1, but then we will use it two more times in Propositions 5.1 and in Lemma 6.6 in order to get bounds on $\alpha_{\epsilon}^{-1} u_{\epsilon}-P U_{x_{\epsilon}, \lambda_{\epsilon}}+$ $\lambda^{-1 / 2}\left(H_{a}\left(x_{\epsilon}, \cdot\right)-H_{0}\left(x_{\epsilon}, \cdot\right)\right)$ and $\alpha_{\epsilon}^{-1} u_{\epsilon}-P U_{x_{\epsilon}, \lambda_{\epsilon}}+\lambda^{-1 / 2} \Pi_{x, \lambda}^{\perp}\left(H_{a}\left(x_{\epsilon}, \cdot\right)-H_{0}\left(x_{\epsilon}, \cdot\right)\right)$, respectively. After the last step we are able to compute the energy to within $o\left(\epsilon^{2}\right)$. We emphasize that in principle there is nothing preventing us from continuing this procedure and computing the energy to even higher precision.

Let us briefly comment on a surprising technical subtlety in our proof. While Theorem 1.7 says that almost minimizers are essentially given by

$$
P U_{x, \lambda}-\lambda^{-1 / 2} \Pi_{x, \lambda}^{\perp}\left(H_{a}(x, \cdot)-H_{0}(x, \cdot)\right)
$$

with $x \in \mathcal{N}_{a}(V)$ a maximum point for the right side in (1.6) and $\lambda$ proportional to $\epsilon^{-1}$, to prove the upper bound we use the simpler functions

$$
P U_{x, \lambda}-\lambda^{-1 / 2}\left(H_{a}(x, \cdot)-H_{0}(x, \cdot)\right)
$$

(with the same choices of $x$ and $\lambda$ ). The difference between the two functions, namely

$$
-\lambda^{-1 / 2} \Pi_{x, \lambda}\left(H_{a}(x, \cdot)-H_{0}(x, \cdot)\right),
$$

can be shown to be of order $\epsilon$ (when $\lambda$ is proportional to $\epsilon^{-1}$ ), but not smaller; see Remark 6.2. Therefore it is not at all obvious that the two families of functions lead to the same (within $o\left(\epsilon^{2}\right)$ ) value of $\mathcal{S}_{a+\epsilon V}[\cdot]$. The fact that they do is contained in Lemma 6.3, where the contributions of $-\lambda^{-1 / 2} \Pi_{x, \lambda}\left(H_{a}(x, \cdot)-H_{0}(x, \cdot)\right)$ to the numerator and to the denominator are shown to cancel each other to within $o\left(\epsilon^{2}\right)$.

At first sight, the problem considered in this paper resembles the problem of minimizing the quotient $\int_{\mathbb{R}^{N}}\left(|\nabla u|^{p}+\epsilon V|u|^{p}\right) d x / \int_{\mathbb{R}^{N}}|u|^{p} d x$ for $p \leq N$, which is a classical problem for $p=2$ [28] motivated by quantum mechanics and which was studied in [14] for 
general $p$. The underlying mechanism, however, is rather different. In these works almost minimizers spread out, whereas here and in its higher dimensional version [18] they concentrate. The concentration regime is much more sensitive to the local details of the perturbation and necessitates, in particular, the use of orthogonality conditions in $T_{x, \lambda}^{\perp}$ and the resulting coercivity.

\subsection{Notation}

Given a set $M$ and two functions $f_{1}, f_{2}: M \rightarrow \mathbb{R}$, we write $f_{1}(m) \lesssim f_{2}(m)$ if there is a numerical constant $c$ such that $f_{1}(m) \leq c f_{2}(m)$ for all $m \in M$. The symbol $\gtrsim$ is defined analogously. For any $p \in[1, \infty]$ and $u \in L^{p}(\Omega)$ we denote

$$
\|u\|_{p}=\|u\|_{L^{p}(\Omega)} .
$$

If $p=2$, we typically drop the subscript and write $\|u\|=\|u\|_{L^{2}(\Omega)}$.

\section{Upper bound on $S(a+\epsilon V)$}

Recall that we always work under Assumption 1.2. In this section (and only in this section), however, we do not assume (1.4).

\subsection{Statement of the bounds and consequences}

Our goal in this section is to prove an upper bound on $S(a+\epsilon V)$ by evaluating the quotient $\mathcal{S}_{a+\epsilon V}[\cdot]$ on a certain family of trial functions. For $x \in \Omega$ and $\lambda>0$, let

$$
\psi_{x, \lambda}(y):=P U_{x, \lambda}(y)-\lambda^{-1 / 2}\left(H_{a}(x, y)-H_{0}(x, y)\right)
$$

This function belongs to $H_{0}^{1}(\Omega)$. We shall prove the following expansions.

Theorem 2.1 As $\lambda \rightarrow \infty$, uniformly for $x$ in compact subsets of $\Omega$ and for $\epsilon \geq 0$,

$$
\begin{aligned}
& \int_{\Omega}\left(\left|\nabla \psi_{x, \lambda}\right|^{2}+(a+\epsilon V) \psi_{x, \lambda}^{2}\right) d y \\
& =3\left(\frac{S}{3}\right)^{\frac{3}{2}}-4 \pi \phi_{a}(x) \lambda^{-1}+2 \pi(4-\pi) a(x) \lambda^{-2}+\frac{\varepsilon}{\lambda} Q_{V}(x) \\
& \quad+o\left(\lambda^{-2}\right)+o\left(\epsilon \lambda^{-1}\right)
\end{aligned}
$$

and

$$
\int_{\Omega} \psi_{x, \lambda}^{6} d y=\left(\frac{S}{3}\right)^{\frac{3}{2}}-8 \pi \phi_{a}(x) \lambda^{-1}+8 \pi a(x) \lambda^{-2}+15 \pi^{2} \phi_{a}(x)^{2} \lambda^{-2}+o\left(\lambda^{-2}\right) .
$$


In particular,

$$
\begin{aligned}
\mathcal{S}_{a+\epsilon V}\left[\psi_{x, \lambda}\right]= & S+\left(\frac{S}{3}\right)^{-\frac{1}{2}} 4 \pi \phi_{a}(x) \lambda^{-1} \\
& +\left(\frac{S}{3}\right)^{-\frac{1}{2}}\left(\frac{\varepsilon}{\lambda} Q_{V}(x)-2 \pi^{2} a(x) \lambda^{-2}-\left(15 \pi^{2}-128\right) \phi_{a}(x)^{2} \lambda^{-2}\right) \\
& +o\left(\lambda^{-2}\right)+o\left(\varepsilon \lambda^{-1}\right) .
\end{aligned}
$$

In the proof of Theorem 2.1 we do not use the fact that $a$ is critical. We only use the fact that $-\Delta+a$ is coercive. In the following corollary we use criticality.

Corollary 2.2 One has $\phi_{a}(x) \geq 0$ for all $x \in \Omega$ and $a(x) \leq 0$ for all $x \in \mathcal{N}_{a}$.

The first part of this corollary appears in [6, Thm. 7]. Note that the second part is non-trivial since we do not assume (1.4).

Proof We apply (2.4) with $\epsilon=0$. We get $\mathcal{S}_{a}\left[\psi_{x, \lambda}\right]=S+(S / 3)^{-1 / 2} 4 \pi \phi_{a}(x) \lambda^{-1}+o\left(\lambda^{-1}\right)$ for any fixed $x \in \Omega$. Since $S=S(a) \leq \mathcal{S}_{a}\left[\psi_{x, \lambda}\right]$, we infer that $\phi_{a}(x) \geq 0$ for all $x \in \Omega$. Similarly, $\mathcal{S}_{a}\left[\psi_{x, \lambda}\right]=S-(S / 3)^{-1 / 2} 2 \pi^{2} a(x) \lambda^{-2}+o\left(\lambda^{-2}\right)$ for any fixed $x \in \mathcal{N}_{a}$ implies that $a(x) \leq 0$ for all $x \in \mathcal{N}_{a}$.

Corollary 2.3 Assume that $\mathcal{N}_{a}(V) \neq \emptyset$. Then $S(a+\epsilon V)<S$ for all $\epsilon>0$ and, as $\epsilon \rightarrow 0+$,

$$
S(a+\epsilon V) \leq S-\left(\frac{S}{3}\right)^{-\frac{1}{2}} \frac{1}{8 \pi^{2}} \sup _{x \in \mathcal{N}_{a}(V)} \frac{Q_{V}(x)^{2}}{|a(x)|} \epsilon^{2}+o\left(\epsilon^{2}\right),
$$

where the right side is to be understood as $-\infty$ if $a(x)=0$ for some $x \in \mathcal{N}_{a}(V)$.

Proof We fix $x \in \mathcal{N}_{a}$ and $k>0$ and apply (2.4) with $\lambda=(k \epsilon)^{-1}$. Since $S(a+\epsilon V) \leq$ $\mathcal{S}_{a}\left[\psi_{x, \lambda}\right]$, we obtain

$$
\limsup _{\epsilon \rightarrow 0} \frac{S(a+\epsilon V)-S}{\epsilon^{2}} \leq(S / 3)^{-1 / 2}\left(k \int_{\Omega} V G_{a}^{2}(x, y) d y-2 \pi^{2} a(x) k^{2}\right) .
$$

Thus,

$$
\limsup _{\epsilon \rightarrow 0} \frac{S(a+\epsilon V)-S}{\epsilon^{2}} \leq(S / 3)^{-1 / 2} \inf _{x \in \mathcal{N}_{a}, k>0}\left(k \int_{\Omega} V G_{a}^{2}(x, y) d y-2 \pi^{2} a(x) k^{2}\right),
$$

which implies the claimed upper bound.

For each $u \in H_{0}^{1}(\Omega), \epsilon \mapsto \mathcal{S}_{a+\epsilon V}[u]$ is an affine linear function, and therefore its infimum over $u$, which is $\epsilon \mapsto S(a+\epsilon V)$, is concave. Since $S(a+\epsilon V)<S$ for all sufficiently small $\epsilon>0$, as we have just shown, we conclude that $S(a+\epsilon V)<S$ for all $\epsilon>0$.

\subsection{Auxiliary facts}

In this preliminary subsection we collect some expansions that will be useful in the proof of Theorem 2.1 as well as later on. In order to emphasize that criticality is not needed, we state them for a function $b \in C(\bar{\Omega}) \cap C^{1}(\Omega)$ such that the operator $-\Delta+b$ in $\Omega$ with Dirichlet boundary conditions is coercive. 
Lemma 2.4 As $\lambda \rightarrow \infty$, uniformly in $x$ from compact subsets of $\Omega$,

$$
\begin{aligned}
\left\|\left(U_{x, \lambda}-\lambda^{-1 / 2} H_{b}(x, \cdot)\right)-\lambda^{-1 / 2} G_{b}(x, \cdot)\right\|_{6 / 5} & =\mathcal{O}\left(\lambda^{-2}\right), \\
\left\|\left(U_{x, \lambda}-\lambda^{-1 / 2} H_{b}(x, \cdot)\right)^{2}-\lambda^{-1} G_{b}(x, \cdot)^{2}\right\|_{1} & =\mathcal{O}\left(\lambda^{-2} \ln \lambda\right) .
\end{aligned}
$$

Proof Since

$$
\left(U_{x, \lambda}-\lambda^{-1 / 2} H_{b}(x, y)\right)-\lambda^{-1 / 2} G_{b}(x, y)=-\lambda^{-1 / 2}\left(\frac{1}{|x-y|}-\frac{\lambda}{\sqrt{1+\lambda^{2}|x-y|^{2}}}\right),
$$

the first bound follows immediately from

$$
0 \leq \frac{1}{|x-y|}-\frac{\lambda}{\sqrt{1+\lambda^{2}|x-y|^{2}}} \leq \min \left\{\frac{1}{|x-y|}, \frac{1}{2 \lambda^{2}|x-y|^{3}}\right\} .
$$

To prove the second bound, we write

$$
\begin{aligned}
& \left(U_{x, \lambda}-\lambda^{-1 / 2} H_{b}(x, y)\right)^{2}-\lambda^{-1} G_{b}^{2}(x, y) \\
& =-\lambda^{-1}\left(\frac{1}{|x-y|^{2}}-\frac{\lambda^{2}}{1+\lambda^{2}|x-y|^{2}}\right) \\
& \quad+2 \lambda^{-1} H_{b}(x, y)\left(\frac{1}{|x-y|}-\frac{\lambda}{\sqrt{\left.1+\lambda^{2}|x-y|^{2}\right)}}\right) .
\end{aligned}
$$

The last term on the right side can be bounded as before, using the fact that $H_{b}(x, \cdot)$ is uniformly bounded in $L^{\infty}(\Omega)$ for $x$ in compact subsets of $\Omega$, see (2.6) below. The first term on the right side can be bounded using

$$
0 \leq \frac{1}{|x-y|^{2}}-\frac{\lambda^{2}}{1+\lambda^{2}|x-y|^{2}} \leq \min \left\{\frac{1}{|x-y|^{2}}, \frac{1}{\lambda^{2}|x-y|^{4}}\right\} .
$$

This proves the lemma.

Lemma 2.5 As $\lambda \rightarrow \infty$, uniformly for $x$ in compact subsets of $\Omega$,

$$
\int_{\Omega} U_{x, \lambda}^{5} H_{b}(x, y) d y=\frac{4 \pi}{3} \phi_{b}(x) \lambda^{-1 / 2}-\frac{4 \pi}{3} b(x) \lambda^{-3 / 2}+o\left(\lambda^{-3 / 2}\right) .
$$

Proof Step 1 We claim that, with $d(x):=\operatorname{dist}(x, \partial \Omega)$,

$$
\left\|H_{b}(x, \cdot)\right\|_{\infty} \lesssim d(x)^{-1} \text { for all } x \in \Omega
$$

Indeed, since $H_{0}(x, \cdot)$ is harmonic in $\Omega$, the maximum principle implies

$$
\left\|H_{0}(x, \cdot)\right\|_{\infty}=\sup _{y \in \partial \Omega} H_{0}(x, y)=d(x)^{-1} .
$$

In order to deduce (2.6) we note that the resolvent identity implies

$$
H_{b}(x, y)-H_{0}(x, y)=\frac{1}{4 \pi} \int_{\Omega} G_{0}(x, z) b(z) G_{b}(z, y) d z .
$$

The claim now follows from the fact that

$$
\sup _{x, y \in \Omega} \int_{\Omega} G_{0}(x, z) G_{b}(z, y) d z<\infty .
$$


Step 2 We claim that for any $x \in \Omega$ there is a $\xi_{x} \in \mathbb{R}^{3}$ such that

$$
H_{b}(x, y)=H_{b}(x, x)+\xi_{x} \cdot(y-x)-\frac{b(x)}{2}|y-x|+o(|y-x|) \quad \text { as } \quad y \rightarrow x .
$$

The asymptotics are uniform for $x$ from compact subsets of $\Omega$.

To prove this, let

$$
\Psi_{x}(y):=H_{b}(x, y)-H_{b}(x, x)+\frac{b(x)}{2}|y-x| .
$$

Using the equation

$$
\Delta_{y} H_{a}(x, y)+a(y) G_{a}(x, y)=0
$$

as well as the fact that $\Delta|x|=2|x|^{-1}$ as distributions we see that $\Psi_{x}$ is a distributional solution of

$$
-\Delta_{y} \Psi_{x}(y)=F_{x}(y) \text { in } \Omega
$$

where

$$
F_{x}(y):=\frac{b(y)-b(x)}{|x-y|}-b(y) H_{b}(x, y) .
$$

By Step 1 and the assumption $b \in C(\bar{\Omega}) \cap C^{1}(\Omega)$, we have $F_{x} \in L_{\mathrm{loc}}^{\infty}(\Omega)$. In particular, $F_{x} \in L_{\mathrm{loc}}^{p}(\Omega)$ for any $3<p<\infty$ and therefore, by elliptic regularity (see, e.g., [21, Thm. 10.2]), $\Psi_{x} \in C_{\mathrm{loc}}^{1, \alpha}(\Omega)$ for $\alpha=1-3 / p$. Thus, in particular, $\Psi_{x} \in C^{1}(\Omega)$. Inserting the Taylor expansion

$$
\Psi_{x}(y)=\nabla_{y} \Psi_{x}(x) \cdot(y-x)+o(|y-x|) \quad \text { as } \quad y \rightarrow x
$$

into (2.10), we obtain the claim with $\xi_{x}=\nabla_{y} \Psi_{x}(x)$. The uniformity statement follows from the fact that if $x$ is from a compact set $K \subset \Omega$, then there is an open set $\omega$ with $K \subset \omega \subset \bar{\omega} \subset \Omega$ such that the norm of $F_{x}$ in $L^{p}(\omega)$ is uniformly bounded for $x \in K$.

Step 3 We now complete the proof of the lemma. Let $0<\rho \leq d(x)$ and write, using Step 2 ,

$$
\begin{aligned}
\int_{\Omega} U_{x, \lambda}^{5} H_{b}(x, y) d y= & \phi_{b}(x) \int_{B_{\rho}(x)} U_{x, \lambda}^{5} d y+\int_{B_{\rho}(x)} U_{x, \lambda}^{5} \xi_{x} \cdot(y-x) d y \\
& -\frac{b(x)}{2} \int_{B_{\rho}(x)} U_{x, \lambda}^{5}|y-x| d y \\
& +o\left(\int_{B_{\rho}(x)} U_{x, \lambda}^{5}|y-x| d y\right)+\int_{\Omega \backslash B_{\rho}(x)} U_{x, \lambda}^{5} H_{b}(x, y) d y
\end{aligned}
$$

with $\rho \rightarrow 0$ as $\lambda \rightarrow \infty$. Since $x$ belongs to a compact subset of $\Omega$, we have $d(x) \gtrsim 1$, and therefore the bound (2.6) from Step 1 implies

$$
\begin{aligned}
\left|\int_{\Omega \backslash B_{\rho}(x)} U_{x, \lambda}^{5} H_{a}(x, y) d y\right| & \lesssim \int_{\Omega \backslash B_{\rho}(x)} U_{x, \lambda}^{5} d y \leq \lambda^{-1 / 2} 4 \pi \int_{\lambda \rho}^{\infty} \frac{t^{2} d t}{\left(1+t^{2}\right)^{5 / 2}} \\
& =\mathcal{O}\left(\lambda^{-5 / 2} \rho^{-2}\right) .
\end{aligned}
$$


Similarly,

$$
\int_{B_{\rho}(x)} U_{x, \lambda}^{5} d y=\lambda^{-1 / 2} 4 \pi \int_{0}^{\lambda \rho} \frac{t^{2} d t}{\left(1+t^{2}\right)^{5 / 2}}=\frac{4 \pi}{3} \lambda^{-1 / 2}+\mathcal{O}\left(\lambda^{-5 / 2} \rho^{-2}\right)
$$

and

$$
\begin{aligned}
\int_{B_{\rho}(x)} U_{x, \lambda}^{5}|x-y| d y & =4 \pi \lambda^{-\frac{3}{2}}\left(\int_{0}^{\infty} \frac{t^{3} d t}{\left(1+t^{2}\right)^{5 / 2}}-\int_{\rho \lambda}^{\infty} \frac{t^{3} d t}{\left(1+t^{2}\right)^{5 / 2}}\right) \\
& =\frac{8 \pi}{3} \lambda^{-\frac{3}{2}}+\mathcal{O}\left(\lambda^{-5 / 2} \rho^{-1}\right) .
\end{aligned}
$$

Finally, since $U_{x, \lambda}$ is radial about $x$,

$$
\int_{B_{\rho}(x)} U_{x, \lambda}^{5}(y) \xi_{x} \cdot(y-x) d y=0 .
$$

Choosing $\rho \rightarrow 0$ with $\lambda \rho^{2} \rightarrow \infty$ we obtain the conclusion of the lemma.

The argument in Step 2 is the only place in this paper where we use the $C^{1}$ assumption on $a$. Clearly the same proof would work if we only assumed $a \in C^{\alpha}(\Omega)$ for some $\alpha>0$.

Lemma 2.6 As $\lambda \rightarrow \infty$, uniformly for $x$ in compact subsets of $\Omega$,

$$
\int_{\Omega} U_{x, \lambda}^{4} H_{b}(x, y)^{2} d y=\pi^{2} \phi_{b}(x)^{2} \lambda^{-1}+o\left(\lambda^{-1}\right) .
$$

The proof is similar, but simpler than that of Lemma 2.5 and is omitted. We only note that the constant comes from

$$
\int_{\mathbb{R}^{3}} U_{x, \lambda}^{4} d y=4 \pi \lambda^{-1} \int_{0}^{\infty} \frac{t^{2} d t}{\left(1+t^{2}\right)^{2}}=\pi^{2} \lambda^{-1}
$$

Lemma 2.7 As $x \rightarrow \infty$, uniformly for $x$ from compact subsets of $\Omega$,

$$
\int_{\Omega} b(y) U_{x, \lambda}(y)\left(\frac{\lambda^{-\frac{1}{2}}}{|x-y|}-U_{x, \lambda}(y)\right) d y=2 \pi(\pi-2) b(x) \lambda^{-2}+\mathcal{O}\left(\lambda^{-3} \log \lambda\right) .
$$

Proof Let $0<\rho \leq \operatorname{dist}(x, \partial \Omega)$. Since $\frac{\lambda^{-\frac{1}{2}}}{|x-y|}-U_{x, \lambda}(y) \geq 0$ for any $x, y \in \Omega$, the differentiability of $b$ at $x$ implies

$$
\begin{aligned}
& \int_{B_{\rho}(x)} b(y) U_{x, \lambda}(y)\left(\frac{\lambda^{-\frac{1}{2}}}{|x-y|}-U_{x, \lambda}(y)\right) d y \\
& \quad=b(x) \int_{B_{\rho}(x)} U_{x, \lambda}(y)\left(\frac{\lambda^{-\frac{1}{2}}}{|x-y|}-U_{x, \lambda}(y)\right) d y+R_{\lambda}
\end{aligned}
$$

with

$$
\begin{aligned}
\left|R_{\lambda}\right| & \lesssim \int_{B_{\rho}(x)}|x-y| U_{x, \lambda}(y)\left(\frac{\lambda^{-\frac{1}{2}}}{|x-y|}-U_{x, \lambda}(y)\right) d y \\
& \lesssim \lambda^{-3} \int_{0}^{\rho \lambda}\left(\frac{t^{2}}{\sqrt{1+t^{2}}}-\frac{t^{3}}{1+t^{2}}\right) d t=\mathcal{O}\left(\lambda^{-3} \ln (\lambda \rho)\right)
\end{aligned}
$$


Moreover,

$$
\begin{aligned}
\int_{B_{\rho}(x)} U_{x, \lambda}(y)\left(\frac{\lambda^{-\frac{1}{2}}}{|x-y|}-U_{x, \lambda}(y)\right) d y & =\lambda^{-2} 4 \pi \int_{0}^{\rho \lambda}\left(\frac{t}{\sqrt{1+t^{2}}}-\frac{t^{2}}{1+t^{2}}\right) d t \\
& =\lambda^{-2} 2 \pi(\pi-2)\left(1+\mathcal{O}\left((\lambda \rho)^{-1}\right)\right) .
\end{aligned}
$$

On the complement of $B_{\rho}(x)$ we use the bound (2.5), which gives

$\left|\int_{\Omega \backslash B_{\rho}(x)} b(y) U_{x, \lambda}(y)\left(\frac{\lambda^{-\frac{1}{2}}}{|x-y|}-U_{x, \lambda}(y)\right) d y\right| \lesssim \lambda^{-2} \int_{\rho \lambda}^{\infty} \frac{d t}{t\left(1+t^{2}\right)^{1 / 2}}=\mathcal{O}\left(\rho^{-1} \lambda^{-3}\right)$.

Choosing $\rho=1 / \ln \lambda$ we obtain the bound in the lemma.

The same proof shows that if $b$ is merely continuous, but not necessarily $C^{1}$, then the expansion still holds with an error $o\left(\lambda^{-2}\right)$. This would be sufficient for our analysis.

\subsection{Expansion of the numerator}

One easily checks that for all $x \in \mathbb{R}^{3}$ and $\lambda>0$,

$$
-\Delta U_{x, \lambda}=3 U_{x, \lambda}^{5} \text {. }
$$

This, together with the Eq. (2.11), the harmonicity of $H_{0}(x, \cdot)$ and (1.9), implies that

$$
-\Delta_{y} \psi_{x, \lambda}(y)=-\Delta_{y} U_{x, \lambda}(y)+\lambda^{-\frac{1}{2}} \Delta_{y} H_{a}(x, y)=3 U_{x, \lambda}^{5}(y)-\lambda^{-\frac{1}{2}} a(y) G_{a}(x, y) \text {. }
$$

We now introduce $f_{x, \lambda}$ by

$$
P U_{x, \lambda}=U_{x, \lambda}-\lambda^{-1 / 2} H_{0}(x, \cdot)-f_{x, \lambda},
$$

and recall that $[24$, Prop. $1(\mathrm{~b})]$, with $d:=\operatorname{dist}(x, \partial \Omega)$,

$$
\left\|f_{x, \lambda}\right\|_{\infty}=\mathcal{O}\left(\lambda^{-5 / 2} d^{-3}\right) .
$$

Hence, by (2.16) and the fact that $\psi_{x, \lambda}$ vanishes on the boundary,

$$
\begin{aligned}
\int_{\Omega}\left|\nabla \psi_{x, \lambda}\right|^{2}= & \int_{\Omega}\left(3 U_{x, \lambda}^{5}(y)-\lambda^{-\frac{1}{2}} a(y) G_{a}(x, y)\right)\left(U_{x, \lambda}(y)-\lambda^{-\frac{1}{2}} H_{a}(x, y)-f_{x, \lambda}(y)\right) d y \\
= & 3 \int_{\Omega} U_{x, \lambda}^{6}(y) d y-3 \lambda^{-\frac{1}{2}} \int_{\Omega} U_{x, \lambda}^{5}(y) H_{a}(x, y) d y \\
& -\lambda^{-1 / 2} \int_{\Omega} a(y) G_{a}(x, y)\left(U_{x, \lambda}(y)-\lambda^{-\frac{1}{2}} H_{a}(x, y)\right) d y \\
& -\int_{\Omega}\left(3 U_{x, \lambda}^{5}(y)-\lambda^{-\frac{1}{2}} a(y) G_{a}(x, y)\right) f_{x, \lambda}(y) d y .
\end{aligned}
$$

It is easy to see that

$$
\int_{\Omega}\left|3 U_{x, \lambda}^{5}(y)-\lambda^{-\frac{1}{2}} a(y) G_{a}(x, y)\right| d y=\mathcal{O}\left(\lambda^{-1 / 2}\right)
$$

and therefore, by (2.18) and the fact that $x$ is in a compact subset of $\Omega$,

$$
\int_{\Omega}\left(3 U_{x, \lambda}^{5}(y)-\lambda^{-\frac{1}{2}} a(y) G_{a}(x, y)\right) f_{x, \lambda}(y) d y=\mathcal{O}\left(\lambda^{-3}\right) .
$$


A simple computation shows that the first term on the right side of (2.19) is

$$
\int_{\Omega} U_{x, \lambda}^{6} d y=\int_{\mathbb{R}^{n}} U_{x, \lambda}^{6} d y+\mathcal{O}\left(\lambda^{-3}\right)=\left(\frac{S}{3}\right)^{\frac{3}{2}}+\mathcal{O}\left(\lambda^{-3}\right) .
$$

For the second term we use Lemma 2.5 and obtain

$$
3 \lambda^{-\frac{1}{2}} \int_{\Omega} U_{x, \lambda}^{5}(y) H_{a}(x, y) d y=4 \pi \phi_{a}(x) \lambda^{-1}-4 \pi a(x) \lambda^{-2}+o\left(\lambda^{-2}\right) .
$$

We will combine the third term with the term coming from $\int_{\Omega} a \psi_{x, \lambda}^{2} d y$.

Using again expansion (2.17) of $P U_{x, \lambda}$ we find

$$
\begin{aligned}
& \int_{\Omega}(a+\epsilon V) \psi_{x, \lambda}^{2}(y) d y=\int_{\Omega}(a+\epsilon V)\left(U_{x, \lambda}-\lambda^{-1 / 2} H_{a}(x, y)\right)^{2} d y \\
& -2 \int_{\Omega}(a+\epsilon V)\left(U_{x, \lambda}-\lambda^{-1 / 2} H_{a}(x, y)\right) f_{x, \lambda} d y+\int_{\Omega}(a+\epsilon V) f_{x, \lambda}^{2} d y .
\end{aligned}
$$

Using (2.18) and the fact that $x$ is in a compact subset of $\Omega$ it is easy to see that

$$
-2 \int_{\Omega}(a+\epsilon V)\left(U_{x, \lambda}-\lambda^{-1 / 2} H_{a}(x, y)\right) f_{x, \lambda} d y+\int_{\Omega}(a+\epsilon V) f_{x, \lambda}^{2} d y=\mathcal{O}\left(\lambda^{-3}(1+\epsilon)\right) .
$$

To summarize, we have shown that

$$
\begin{aligned}
\int_{\Omega}\left(\left|\nabla \psi_{x, \lambda}\right|^{2}+a \psi_{x, \lambda}^{2}\right) d y= & 3\left(\frac{S}{3}\right)^{\frac{3}{2}}-4 \pi \phi_{a}(x) \lambda^{-1}+4 \pi a(x) \lambda^{-2}+T(x, \lambda) \\
& +\epsilon \int_{\Omega} V\left(U_{x, \lambda}-\lambda^{-1 / 2} H_{a}(x, y)\right)^{2} d y+o\left(\lambda^{-2}\right)+\mathcal{O}\left(\epsilon \lambda^{-3}\right)
\end{aligned}
$$

with

$$
T(x, \lambda):=\int_{\Omega} a(y)\left(U_{x, \lambda}(y)-\lambda^{-1 / 2} H_{a}(x, y)\right)\left(U_{x, \lambda}(y)-\frac{\lambda^{-1 / 2}}{|x-y|}\right) d y .
$$

Similarly as in the proof of Lemma 2.7 one finds that

$$
\lambda^{-1 / 2} \int_{\Omega} a(y) H_{a}(x, y)\left(\frac{\lambda^{-1 / 2}}{|x-y|}-U_{x, \lambda}(y)\right) d y=\mathcal{O}\left(\lambda^{-3} \ln \lambda\right) .
$$

Hence, by Lemma 2.7,

$$
T(x, \lambda)=-2 \pi(\pi-2) a(x) \lambda^{-2}+o\left(\lambda^{-2}\right) .
$$

Finally, by Lemma 2.4,

$$
\int_{\Omega} V\left(U_{x, \lambda}-\lambda^{-1 / 2} H_{a}(x, y)\right)^{2} d y=\lambda^{-1} \int_{\Omega} V G_{a}(x, y)^{2} d y+\mathcal{O}\left(\lambda^{-2} \ln \lambda\right) .
$$

This proves the first assertion in Theorem 2.1. 


\subsection{Expansion of the denominator}

By the decomposition (2.17) for $P U_{x, \lambda}$ we obtain

$$
\int_{\Omega} \psi_{x, \lambda}^{6} d y=\int_{\Omega}\left(U_{x, \lambda}-\lambda^{-1 / 2} H_{a}(x, y)\right)^{6} d y+\mathcal{O}\left(\left\|U_{x, \lambda}-\lambda^{-1 / 2} H_{a}(x, \cdot)\right\|_{5}^{5}\left\|f_{x, \lambda}\right\|_{\infty}+\left\|f_{x, \lambda}\right\|_{6}^{6}\right) .
$$

Using (2.6) and (2.18), together with the fact that $x$ is in a compact subset of $\Omega$, we see that the remainder term is $\mathcal{O}\left(\lambda^{-3}\right)$. Next, we expand

$$
\begin{aligned}
& \int_{\Omega}\left(U_{x, \lambda}-\lambda^{-1 / 2} H_{a}(x, y)\right)^{6} d y \\
& \quad=\int_{\Omega} U_{x, \lambda}^{6} d y-6 \lambda^{-1 / 2} \int_{\Omega} U_{x, \lambda}^{5} H_{a}(x, y) d y+15 \lambda^{-1} \int_{\Omega} U_{x, \lambda}^{4} H_{a}(x, y)^{2} d y \\
& \quad+\mathcal{O}\left(\lambda^{-3 / 2}\left\|U_{x, \lambda}\right\|_{3}^{3}\left\|H_{a}(x, \cdot)\right\|_{\infty}^{2}+\lambda^{-3}\left\|H_{a}(x, \cdot)\right\|_{6}^{6}\right) .
\end{aligned}
$$

Using (2.6), together with the fact that $x$ is in a compact subset of $\Omega$, we see that the remainder term is $\mathcal{O}\left(\lambda^{-3} \ln \lambda\right)$. The first three terms on the right side are evaluated in (2.20) and Lemmas 2.5 and 2.6. This proves the second assertion in Theorem 2.1.

\subsection{Expansion of the quotient}

Expansion (2.3) implies that

$$
\begin{aligned}
\left(\int_{\Omega} \psi_{x, \lambda}^{6} d y\right)^{-1 / 3}= & \left(\frac{S}{3}\right)^{-\frac{1}{2}}+\left(\frac{S}{3}\right)^{-2} \frac{8 \pi}{3} \phi_{a}(x) \lambda^{-1} \\
& +\left(\frac{S}{3}\right)^{-2}\left(-\frac{8 \pi}{3} a(x)-5 \pi^{2} \phi_{a}(x)^{2}+\frac{2}{9} \frac{64 \pi^{2}}{(S / 3)^{3 / 2}} \phi_{a}(x)^{2}\right) \lambda^{-2} \\
& +o\left(\lambda^{-2}\right) .
\end{aligned}
$$

Expansion (2.4) now follows by multiplying the previous equation with (2.2). This concludes the proof of Theorem 2.1 .

\section{Lower bound on $S(a+\epsilon V)$ : preliminaries}

\subsection{The asymptotic form of almost minimizers}

The remainder of this paper is concerned with proving a lower bound on $S(a+\epsilon V)$ that matches the upper bound from Corollary 2.3. We will establish this by proving that functions $u_{\epsilon}$ for which $\mathcal{S}_{a+\epsilon V}\left[u_{\epsilon}\right]$ is 'close' to $S(a+\epsilon V)$ are 'close' to the functions $\psi_{x, \lambda}$ used in the upper bound for certain $x$ and $\lambda$ depending on $\epsilon$. We will prove this in several steps. The very first step is the following proposition.

Proposition 3.1 Let $\left(u_{\epsilon}\right) \subset H_{0}^{1}(\Omega)$ be a sequence of functions satisfying

$$
\mathcal{S}_{a+\epsilon V}\left[u_{\epsilon}\right]=S+o(1), \quad \int_{\Omega} u_{\epsilon}^{6} d x=(S / 3)^{3 / 2} .
$$


Then, along a subsequence,

$$
u_{\epsilon}=\alpha_{\epsilon}\left(P U_{x_{\epsilon}, \lambda_{\epsilon}}+w_{\epsilon}\right) \text {, }
$$

where

$$
\begin{aligned}
\alpha_{\epsilon} & \rightarrow s \text { for some } s \in\{-1,+1\}, \\
x_{\epsilon} & \rightarrow x_{0} \text { for some } x_{0} \in \bar{\Omega}, \\
\lambda_{\epsilon} d_{\epsilon} & \rightarrow \infty \\
\left\|\nabla w_{\epsilon}\right\| & \rightarrow 0 \text { and } w_{\epsilon} \in T_{x_{\epsilon}, \lambda_{\epsilon}}^{\perp} .
\end{aligned}
$$

Here $d_{\varepsilon}=\operatorname{dist}\left(x_{\varepsilon}, \partial \Omega\right)$.

If the $u_{\epsilon}$ are minimizers for $S(a+\epsilon V)$, and therefore solutions to the corresponding Euler-Lagrange equation, this proposition is well-known and goes back to work of Struwe [29] and Bahri-Coron [5]. The result for almost minimizers is also well-known to specialists, but since we have not been able to find a proof in the literature, we include one in Appendix B. Here we only emphasize that the fact that $u_{\epsilon}$ converges weakly to zero in $H_{0}^{1}(\Omega)$ is deduced from a theorem of Druet [12] which says that $S(a)$ is not attained for critical $a$. (Note that this part of the paper [12] is valid for $a \in L^{3 / 2}(\Omega)$, without any further regularity requirement.)

Convention From now on we will assume that

$$
S(a+\epsilon V)<S \text { for all } \epsilon>0
$$

and that $\left(u_{\epsilon}\right)$ satisfies (1.10). In particular, assumption (3.1) is satisfied. We will always work with a sequence of $\epsilon$ 's for which the conclusions of Proposition 3.1 hold. To enhance readability, we will drop the index $\epsilon$ from $\alpha_{\epsilon}, x_{\epsilon}, \lambda_{\epsilon}, d_{\epsilon}$ and $w_{\epsilon}$.

\section{A priori bounds}

\subsection{Statement of the bounds}

From Proposition 3.1 we know that $\|\nabla w\|=o(1)$ and that the limit point $x_{0}$ of $\left(x_{\epsilon}\right)$ lies in $\bar{\Omega}$. The following proposition, which is the main result of this section, improves both these results.

Proposition 4.1 As $\epsilon \rightarrow 0$,

$$
\begin{aligned}
& \|\nabla w\|=\mathcal{O}\left(\lambda^{-1 / 2}\right), \\
& d^{-1}=\mathcal{O}(1)
\end{aligned}
$$

and

$$
\lambda(S-S(a+\epsilon V))=\mathcal{O}(1) \text { and } \lambda\left(\mathcal{S}_{a+\epsilon V}\left[u_{\epsilon}\right]-S(a+\epsilon V)\right)=o(1) .
$$

The bounds (4.1) and (4.2) were shown in [15, Lem. 2.2 and Thm. 1.1] in the case where $u_{\epsilon}$ is a minimizer for $S(a+\epsilon V)$. Since the proof in [15] uses the Euler-Lagrange equation satisfied by minimizers, this proof is not applicable in our case. We will replace the use of the Euler-Lagrange equation by a suitable expansion of $\mathcal{S}_{a+\epsilon V}\left[u_{\epsilon}\right]$, which is carried out in Sect. 4.2. The other ingredient in the proof of [15, Lem. 2.2] and in our proof is the coercivity of a certain quadratic form, see Lemma 4.3 in Sect. 4.3. Finally, in Sect. 4.4 we will prove Proposition 4.1. 


\subsection{A first expansion}

In this subsection, we shall prove the following lemma.

Lemma 4.2 As $\epsilon \rightarrow 0$,

$$
\begin{aligned}
\mathcal{S}_{a+\epsilon V}\left[u_{\varepsilon}\right]= & S+(S / 3)^{-1 / 2} 4 \pi \phi_{0}(x) \lambda^{-1}+(S / 3)^{-1 / 2} \int_{\Omega}\left(|\nabla w|^{2}+a w^{2}-15 U_{x, \lambda}^{4} w^{2}\right) d y \\
& +O\left(\lambda^{-1 / 2}\|\nabla w\|\right)+o\left((d \lambda)^{-1}\right)+o\left(\|\nabla w\|^{2}\right) .
\end{aligned}
$$

Proof of Lemma 4.2 We will expand separately the numerator and the denominator in $\mathcal{S}_{a+\epsilon V}\left[u_{\varepsilon}\right]$.

Expansion of the numerator Since $w$ is orthogonal to $P U$, we have

$$
\alpha^{-2} \int_{\Omega}\left|\nabla u_{\epsilon}\right|^{2} d y=\int_{\Omega}\left|\nabla P U_{x, \lambda}\right|^{2} d y+\int_{\Omega}|\nabla w|^{2} d y .
$$

The first term on the right side is computed in (A.1). The other terms in the numerator are

$$
\alpha^{-2} \int_{\Omega}(a+\epsilon V) u_{\epsilon}^{2} d y=\int_{\Omega}(a+\epsilon V) P U_{\lambda, x}^{2} d y+2 \int_{\Omega}(a+\varepsilon) P U_{\lambda, x} w d y+\int_{\Omega}(a+\epsilon V) w^{2} d y .
$$

Since $0 \leq P U_{x, \lambda} \leq U_{x, \lambda} \leq \lambda^{-1 / 2}|x-y|^{-1}$, see [24, Prop. 1], we have

$$
\left|\int_{\Omega}(a+\epsilon V) P U_{x, \lambda}^{2} d y\right| \leq\|a+\epsilon V\|_{\infty} \lambda^{-1} \int_{\Omega} \frac{d y}{|x-y|^{2}}=\mathcal{O}\left(\lambda^{-1}\right) .
$$

Clearly,

$$
\epsilon\left|\int_{\Omega} V w^{2} d y\right| \leq \epsilon\|V\|_{\infty}\|w\|^{2} \lesssim \epsilon\|V\|_{\infty}\|\nabla w\|^{2}=o\left(\|\nabla w\|^{2}\right),
$$

and, by (A.5),

$$
\left|\int_{\Omega}(a+\epsilon V) P U_{x, \lambda} w d x\right| \leq\|a+\epsilon V\|_{\infty}\left\|P U_{x, \lambda}\right\|_{6 / 5}\|w\|_{6}=\mathcal{O}\left(\lambda^{-1 / 2}\|\nabla w\|\right) .
$$

To summarize, the numerator is $\alpha^{2}$ times

$$
3^{-1 / 2} S^{3 / 2}-4 \pi \phi_{0}(x) \lambda^{-1}+\int_{\Omega}\left(|\nabla w|^{2}+a w^{2}\right) d y+\mathcal{O}\left(\lambda^{-1 / 2}\|\nabla w\|\right)+o\left((\lambda d)^{-1}\right)+o\left(\|\nabla w\|^{2}\right) .
$$

Expansion of the denominator We have

$$
\alpha^{-6} \int_{\Omega} u_{\epsilon}^{6} d y=\int_{\Omega} P U_{x, \lambda}^{6} d y+6 \int_{\Omega} P U_{x, \lambda}^{5} w d y+15 \int_{\Omega} P U_{x, \lambda}^{4} w^{2} d y+\mathcal{O}\left(\|\nabla w\|^{3}\right) .
$$

The first term on the right side is computed in (A.2). Moreover, abbreviating $\phi_{x, \lambda}:=$ $\lambda^{-1 / 2} H_{0}(x, \cdot)+f_{x, \lambda}$, so that, by (2.17), $P U_{x, \lambda}=U_{x, \lambda}-\phi_{x, \lambda}$, we find

$$
\int_{\Omega} P U_{x, \lambda}^{5} w d y=\int_{\Omega} U_{x, \lambda}^{5} w d y+\mathcal{O}\left(\int_{\Omega} U_{x, \lambda}^{4} \phi_{x, \lambda}|w| d y+\int_{\Omega} \phi_{x, \lambda}^{5}|w| d y\right) .
$$

(Note that $\phi_{x, \lambda} \geq 0$, since $P U_{x, \lambda} \leq U_{x, \lambda}$ by [24, Prop. 1 (a)].) By (2.15), (1.9), the fact that $w$ vanishes on the boundary and since $w \in T_{x, \lambda}^{\perp}$, we have

$$
\int_{\Omega} U_{x, \lambda}^{5} w d y=\frac{1}{3} \int_{\Omega}\left(-\Delta U_{x, \lambda}\right) w d y=\frac{1}{3} \int_{\Omega} \nabla P U_{x, \lambda} \cdot \nabla w d y=0 .
$$


Also, by the equation after [15, (10)],

$$
\int_{\Omega} U_{x, \lambda}^{4} \phi_{x, \lambda}|w| d y+\int_{\Omega} \phi_{x, \lambda}^{5}|w| d y=\mathcal{O}\left((d \lambda)^{-1}\|\nabla w\|\right)=o\left((d \lambda)^{-1}\right) .
$$

Finally,

$$
\int_{\Omega} P U_{x, \lambda}^{4} w^{2} d y=\int_{\Omega} U_{x, \lambda}^{4} w^{2} d y+\mathcal{O}\left(\int_{\Omega} U_{x, \lambda}^{3} \phi_{x, \lambda} w^{2} d y+\int_{\Omega} \phi_{x, \lambda}^{4} w^{2} d y\right)
$$

and, since $\left\|\phi_{x, \lambda}\right\|_{6}=\mathcal{O}\left((d \lambda)^{-1 / 2}\right)$ by [24, Prop. 1 (c) ],

$$
\int_{\Omega} U_{x, \lambda}^{3} \phi_{x, \lambda} w^{2} d y+\int_{\Omega} \phi_{x, \lambda}^{4} w^{2} d y=o\left(\|\nabla w\|^{2}\right) .
$$

To summarize, we have shown that

$$
\alpha^{-6} \int_{\Omega} u_{\epsilon}^{6} d y=(S / 3)^{3 / 2}-8 \pi \phi_{0}(x) \lambda^{-1}+15 \int_{\Omega} U_{x, \lambda}^{4} w^{2} d y+o\left((d \lambda)^{-1}\right)+o\left(\|\nabla w\|^{2}\right)
$$

and therefore, by the rough bound $\int_{\Omega} U_{x, \lambda} w^{2} d y \leq\left\|U_{x, \lambda}\right\|_{6}^{4}\|w\|_{6}^{2} \lesssim\left\|U_{x, \lambda}\right\|_{6}^{4}\|\nabla w\|^{2}=o(1)$,

$$
\begin{aligned}
\alpha^{2}\left(\int_{\Omega} u_{\epsilon}^{6} d y\right)^{-1 / 3}= & \left(\frac{S}{3}\right)^{-\frac{1}{2}}+\left(\frac{S}{3}\right)^{-2} \frac{8 \pi}{3} \phi_{0}(x) \lambda^{-1}-45 S^{-2} \int_{\Omega} U_{x, \lambda}^{4} w^{2} d y \\
& +o\left((d \lambda)^{-1}\right)+o\left(\|\nabla w\|^{2}\right) .
\end{aligned}
$$

The lemma follows immediately from the expansions of the numerator and the denominator.

\subsection{Coercivity}

We will frequently use the following bound from [15, Lem. 2.2].

Lemma 4.3 There are constants $T_{*}<\infty$ and $\rho>0$ such that for all $x \in \Omega$, all $\lambda>0$ with $d \lambda \geq T_{*}$ and all $v \in T_{x, \lambda}^{\perp}$,

$$
\int_{\Omega}\left(|\nabla v|^{2}+a v^{2}-15 U_{x, \lambda}^{4} v^{2}\right) d y \geq \rho \int_{\Omega}|\nabla v|^{2} d y .
$$

The proof proceeds by compactness, using the inequality [24, (D.1)]

$$
\int_{\Omega}\left(|\nabla v|^{2}-15 U_{x, \lambda}^{4} v^{2}\right) d y \geq \frac{4}{7} \int_{\Omega}|\nabla v|^{2} d y \quad \text { for all } v \in T_{x, \lambda}^{\perp} .
$$

For details of the proof we refer to [15].

\subsection{Proof of Proposition 4.1}

We combine the expansion from Lemma 4.2 with the coercivity bound from Lemma 4.3 and the fact that $c:=\inf _{y \in \Omega} \operatorname{dist}(y, \partial \Omega) \phi_{0}(y)>0$, see [24, (2.8)] or [16, Lem. 8.3]. (Note that this bound uses the $C^{2}$ assumption on $\partial \Omega$.) Thus,

$$
\begin{aligned}
\mathcal{S}_{a+\epsilon V}\left[u_{\epsilon}\right] & \geq S+\left((S / 3)^{-1 / 2} 4 \pi c+o(1)\right)(d \lambda)^{-1} \\
& +\left((S / 3)^{-1 / 2} \rho+o(1)\right)\|\nabla w\|^{2}+\mathcal{O}\left(\lambda^{-1 / 2}\|\nabla w\|\right) .
\end{aligned}
$$


Since $\lambda^{-1 / 2}\|\nabla w\| \leq \delta\|\nabla w\|^{2}+(4 \delta)^{-1} \lambda^{-1}$ for every $\delta>0$, we obtain, for all sufficiently small $\epsilon>0$ and some constants $c_{1}, c_{2}>0$ and $C<\infty$ independent of $\epsilon$,

$$
C \lambda^{-1}+\left(\mathcal{S}_{a+\epsilon V}\left[u_{\epsilon}\right]-S(a+\epsilon V)\right) \geq S-S(a+\epsilon V)+c_{1}(d \lambda)^{-1}+c_{2}\|\nabla w\|^{2} .
$$

By assumption (1.10), this becomes

$$
C \lambda^{-1} \geq(1+o(1))(S-S(a+\epsilon V))+c_{1}(d \lambda)^{-1}+c_{2}\|\nabla w\|^{2} .
$$

Since all three terms on the right side are non-negative, we obtain (4.1), (4.2) and the first bound in (4.3). The second bound in (4.3) follows from the first one by assumption (1.10). This completes the proof of the proposition.

\section{A priori bounds reloaded}

\subsection{Statement and heuristics for the improved a priori bound}

In order to prove a sufficiently precise lower bound on $S(a+\epsilon V)$ we need more detailed information on the almost minimizers $u_{\varepsilon}$. Here we extract the leading term from the remainder term $w=w_{\varepsilon}$ in (3.2).

Proposition 5.1 One has, as $\epsilon \rightarrow 0$,

$$
\lambda(S-S(a+\epsilon V))=o(1), \quad \phi_{a}(x)=o(1)
$$

and

$$
w=-\lambda^{-1 / 2}\left(H_{a}(x, \cdot)-H_{0}(x, \cdot)\right)+q \text { with }\|\nabla q\|=o\left(\lambda^{-1 / 2}\right) .
$$

Note that the second statement in (5.1) implies that $\phi_{a}\left(x_{0}\right)=0$ for the limit point $x_{0}$ in (3.3). In particular, together with Corollary 2.2 , we obtain $\min _{\Omega} \phi_{a}=0$ for critical $a$, which is Druet's theorem [12]. Our proof, which is closely related to that by Esposito [15], uses another theorem of Druet, which says that $S(a)$ is not attained for critical $a$ [12, Step 1] (see Proposition 3.1), but is otherwise independent of [12].

The proof of Proposition 5.1 is given at the end of this section. Let us explain the heuristics behind the proof. In Lemma 5.2 we will derive the following expansion,

$$
\begin{aligned}
& \mathcal{S}_{a+\epsilon V}\left[u_{\varepsilon}\right]=S+\lambda^{-1}\left(\frac{S}{3}\right)^{-\frac{1}{2}} \\
& \left(4 \pi \phi_{a}(x)+(4 \pi)^{-1} \iint_{\Omega \times \Omega} G_{0}(x, y) a(y) G_{a}\left(y, y^{\prime}\right) a\left(y^{\prime}\right) G_{0}\left(y^{\prime}, x\right) d y d y^{\prime}\right) \\
& +\left(\frac{S}{3}\right)^{-\frac{1}{2}} \int_{\Omega}\left(|\nabla w|^{2}+a w^{2}+2 \lambda^{-1 / 2} a G_{0}(x, y) w-15 U_{x, \lambda}^{4} w^{2}\right) d y+o\left(\lambda^{-1}\right) .
\end{aligned}
$$

Note that this is an improvement over the expansion in Lemma 4.2, which only had a remain$\operatorname{der} \mathcal{O}\left(\lambda^{-1}\right)$. This improvement is possible thanks to the information from Proposition 4.1.

From the expansion (5.3) we want to determine the asymptotic form of $w$. In order to (almost) minimize the quotient $\mathcal{S}_{a+\epsilon V}\left[u_{\varepsilon}\right]$ the function $w$ will (almost) minimize the expression

$$
\int_{\Omega}\left(|\nabla w|^{2}+a w^{2}+2 \lambda^{-1 / 2} a G_{0}(x, y) w-15 U_{x, \lambda}^{4} w^{2}\right) d y .
$$


This is quadratic and linear in $w$, so it can be minimized by 'completing a square'. If the term $-15 U_{x, \lambda}^{4}$ were absent, then the minimum would be

$$
-\lambda^{-1}(4 \pi)^{-1} \iint_{\Omega \times \Omega} G_{0}(x, y) a(y) G_{a}\left(y, y^{\prime}\right) a\left(y^{\prime}\right) G_{0}\left(y^{\prime}, x\right) d y d y^{\prime}
$$

and the optimal choice for $w$ would be $-\lambda^{-1 / 2}\left(H_{a}(x, \cdot)-H_{0}(x, \cdot)\right)$. Using the positive contribution that arises when completing the square, we will be able to show that if $u_{\epsilon}$ almost minimizes $S(a+\epsilon V)$, then $w$ almost minimizes the above problem and is therefore almost equal to $-\lambda^{-1 / 2}\left(H_{a}(x, \cdot)-H_{0}(x, \cdot)\right)$. Proposition 5.1 provides a quantitative version of these heuristics.

As the above argument shows, the main difficulty will be to show that the term $-15 U_{x, \lambda}^{4}$ is negligible to within $o\left(\lambda^{-1}\right)$. This does not follow from a straightforward bound since $\|\nabla w\|^{2}$ is only $\mathcal{O}\left(\lambda^{-1}\right)$. The orthogonality conditions satisfied by $w$ will play an important role.

\subsection{A second expansion}

In this subsection, we shall prove the following lemma.

Lemma 5.2 As $\epsilon \rightarrow 0$,

$$
\begin{aligned}
& \mathcal{S}_{a+\epsilon V}\left[u_{\varepsilon}\right]=S+\lambda^{-1} \\
& \left(\frac{S}{3}\right)^{-\frac{1}{2}}\left(4 \pi \phi_{a}(x)+(4 \pi)^{-1} \iint_{\Omega \times \Omega} G_{0}(x, y) a(y) G_{a}\left(y, y^{\prime}\right) a\left(y^{\prime}\right) G_{0}\left(y^{\prime}, x\right) d y d y^{\prime}\right) \\
& \quad+\left(\frac{S}{3}\right)^{-\frac{1}{2}} \int_{\Omega}\left(|\nabla w|^{2}+a w^{2}+2 \lambda^{-1 / 2} a G_{0}(x, y) w-15 U_{x, \lambda}^{4} w^{2}\right) d y+o\left(\lambda^{-1}\right) .
\end{aligned}
$$

Proof Expansion of the numerator We claim that

$$
\begin{aligned}
& \alpha^{-2} \int_{\Omega}\left(\left|\nabla u_{\varepsilon}\right|^{2}+a u_{\epsilon}^{2}+\epsilon V u_{\epsilon}^{2}\right) d y=3^{-1 / 2} S^{3 / 2}-\lambda^{-1}\left(4 \pi \phi_{0}(x)-\int_{\Omega} a G_{0}(x, y)^{2} d y\right) \\
& \quad+\int_{\Omega}\left(|\nabla w|^{2}+a w^{2}+2 \lambda^{-1 / 2} a G_{0}(x, y) w\right) d y+o\left(\lambda^{-1}\right) .
\end{aligned}
$$

Indeed, arguing as in the proof of Lemma 4.2 and using the bounds on $d$ and $\|\nabla w\|$ from Proposition 4.1, we obtain

$$
\begin{aligned}
\alpha^{-2} \int_{\Omega}\left(\left|\nabla u_{\varepsilon}\right|^{2}+a u_{\epsilon}^{2}+\epsilon V u_{\epsilon}^{2}\right) d y= & 3^{-1 / 2} S^{3 / 2}-4 \pi \phi_{0}(x) \lambda^{-1}+\int_{\Omega} a P U_{x, \lambda}^{2} d y \\
& +\int_{\Omega}\left(|\nabla w|^{2}+a w^{2}+2 a P U_{x, \lambda} w\right) d y+o\left(\lambda^{-1}\right) .
\end{aligned}
$$


Note that here we have kept the term $\int_{\Omega} a\left(P U_{x, \lambda}^{2}+2 P U_{x, \lambda} w\right) d y$ instead of estimating it. We now treat this contribution more carefully. We expand $P U_{x, \lambda}$ as in (2.17), which leads to

$$
\begin{aligned}
\int_{\Omega} a & \left.P U_{x, \lambda}^{2}+2 P U_{x, \lambda} w\right) d y \\
= & \int_{\Omega} a\left(\left(U_{x, \lambda}-\lambda^{-1 / 2} H_{0}(x, y)\right)^{2}+2\left(U_{x, \lambda}-\lambda^{-1 / 2} H_{0}(x, y)\right) w\right) d y \\
& -2 \int_{\Omega} a\left(P U_{x, \lambda}+w\right) f_{x, \lambda} d y-\int_{\Omega} a f_{x, \lambda}^{2} d y .
\end{aligned}
$$

By (2.18) and (A.5), taking into account (4.2),

$$
\begin{aligned}
& \left|\int_{\Omega} a\left(2\left(P U_{x, \lambda}+w\right) f_{x, \lambda}+f_{x, \lambda}^{2}\right) d y\right| \\
& \quad=\mathcal{O}\left(\|a\|_{\infty}\left(\left\|P U_{x, \lambda}\right\|_{6 / 5}\|f\|_{6}+\|w\|_{6}\left\|f_{x, \lambda}\right\|_{6 / 5}+\left\|f_{x, \lambda}\right\|^{2}\right)\right) \\
& \quad=\mathcal{O}\left(\lambda^{-3}\right) .
\end{aligned}
$$

On the other hand, by Lemma 2.4 ,

$$
\begin{gathered}
\int_{\Omega} a\left(\left(U_{x, \lambda}-\lambda^{-1 / 2} H_{0}(x, y)\right)^{2}+2\left(U_{x, \lambda}-\lambda^{-1 / 2} H_{0}(x, y)\right) w\right) d y \\
=\int_{\Omega} a\left(\lambda^{-1} G_{0}(x, y)^{2}+2 \lambda^{-1 / 2} G_{0}(x, y) w\right) d y+\mathcal{O}\left(\lambda^{-2} \ln \lambda\right) .
\end{gathered}
$$

This proves (5.5).

Expansion of the denominator Combining the bound from the proof of Lemma 4.2 with the bounds on $d$ and $\|\nabla w\|$ from Proposition 4.1, we obtain

$\alpha^{2}\left(\int_{\Omega} u_{\epsilon}^{6} d y\right)^{-1 / 3}=(S / 3)^{-1 / 2}+(S / 3)^{-2} \frac{8 \pi}{3} \phi_{0}(x) \lambda^{-1}-45 S^{-2} \int_{\Omega} U_{x, \lambda}^{4} w^{2} d y+o\left(\lambda^{-1}\right)$.

Expansion of the quotient Multiplying (5.5) and (5.6) gives

$$
\begin{aligned}
& \mathcal{S}_{a+\epsilon V}\left[u_{\varepsilon}\right]=S+\lambda^{-1}(S / 3)^{-1 / 2} 4 \pi \phi_{0}(x)+\lambda^{-1}(S / 3)^{-1 / 2} \int_{\Omega} a G_{0}(x, y)^{2} d y \\
& +(S / 3)^{-1 / 2} \int_{\Omega}\left(|\nabla w|^{2}+a w^{2}+2 \lambda^{-1 / 2} a G_{0}(x, y) w-15 U_{x, \lambda}^{4} w^{2}\right) d y+o\left(\lambda^{-1}\right) .
\end{aligned}
$$

The resolvent identity together with the symmetry $G_{0}(x, y)=G_{0}(y, x)$ implies

$$
\begin{aligned}
& \int_{\Omega} a(y) G_{0}(x, y)^{2} d y-(4 \pi)^{-1} \iint_{\Omega \times \Omega} G_{0}(a, y) a(y) G_{a}\left(y, y^{\prime}\right) a\left(y^{\prime}\right) G_{0}\left(y^{\prime}, x\right) d y d y^{\prime} \\
& \quad=\int_{\Omega} G_{0}(x, y) a(y) G_{a}(y, x) d y=4 \pi\left(\phi_{a}(x)-\phi_{0}(x)\right) .
\end{aligned}
$$

This completes the proof of the lemma.

\subsection{Regularization and coercivity}

In this subsection we will show that the coercivity bound from Lemma 4.3 remains essentially true after regularization. A convenient regularization procedure for us is a spectral cut-off. 
Namely, we denote by $\mathbb{1}(-\Delta+a \leq \mu)$ the spectral projection for the interval $(-\infty, \mu]$ of the self-adjoint operator $-\Delta+a$ in $L^{2}(\Omega)$ with Dirichlet boundary condition. The parameter $\mu$ here will be later chosen large depending on $\epsilon$.

Lemma 5.3 Let $v \in H_{0}^{1}(\Omega)$. Then for any $\mu \geq 1$,

$$
\|\mathbb{1}(-\Delta+a \leq \mu) v\|_{\infty} \lesssim \mu^{1 / 4}\|\nabla v\| .
$$

Proof Let $a_{-}=\max \{0,-a\}$. By the maximum principle or the Trotter product formula, we have

$$
0 \leq e^{-t(-\Delta+a)}(x, x) \leq(4 \pi t)^{-3 / 2} e^{t\left\|a_{-}\right\|_{\infty}} \text { for all } t>0 ;
$$

see, e.g., [11, Thm. 2.4.4] for related estimates.

We denote by $E_{n}$ the eigenvalues of $-\Delta+a$ in $L^{2}(\Omega)$ and by $\Phi_{n}$ the corresponding $L^{2}$-normalized eigenfunctions. We bound for any $x \in \Omega$

$$
\begin{aligned}
|(\mathbb{1}(-\Delta+a \leq \mu) v)(x)| & =\left|\sum_{E_{n} \leq \mu}\left(\Phi_{n}, v\right) \Phi_{n}(x)\right| \\
& \leq\left(\sum_{E_{n} \leq \mu} E_{n}\left|\left(\Phi_{n}, v\right)\right|^{2}\right)^{1 / 2}\left(\sum_{E_{n} \leq \mu} E_{n}^{-1}\left|\Phi_{n}(x)\right|^{2}\right)^{1 / 2} .
\end{aligned}
$$

We clearly have

$$
\sum_{E_{n} \leq \mu} E_{n}\left|\left(\Phi_{n}, v\right)\right|^{2} \leq \sum_{n} E_{n}\left|\left(\Phi_{n}, v\right)\right|^{2}=(v,(-\Delta+a) v) \lesssim\|\nabla v\|^{2} .
$$

The heat kernel bound (5.8) implies that for any $s>0$ and $t>0$

$$
\sum_{E_{n} \leq s}\left|\Phi_{n}(x)\right|^{2} \leq e^{t s} \sum_{E_{n} \leq s} e^{-t E_{n}}\left|\Phi_{n}(x)\right|^{2} \leq e^{t\left(s+\left\|a_{-}\right\|_{\infty}\right)}(4 \pi t)^{-3 / 2},
$$

and choosing $t=(3 / 2)\left(s+\left\|a_{-}\right\|_{\infty}\right)^{-1}$ we obtain for any $s>0$,

$$
\sum_{E_{n} \leq s}\left|\Phi_{n}(x)\right|^{2} \leq\left(\frac{e}{6 \pi}\right)^{3 / 2}\left(s+\left\|a_{-}\right\|_{\infty}\right)^{3 / 2} .
$$

Thus, writing $E^{-1}=\int_{E}^{\infty} s^{-2} d s$, we get

$$
\begin{aligned}
\sum_{E_{n} \leq \mu} E_{n}^{-1}\left|\Phi_{n}(x)\right|^{2} & =\int_{0}^{\infty} \sum_{E_{n} \leq \mu}\left|\Phi_{n}(x)\right|^{2} \mathbb{1}\left(E_{n} \leq s\right) \frac{d s}{s^{2}}=\int_{E_{1}}^{\infty} \sum_{E_{n} \leq \min \{\mu, s\}}\left|\Phi_{n}(x)\right|^{2} \frac{d s}{s^{2}} \\
& \leq\left(\frac{e}{6 \pi}\right)^{3 / 2} \int_{E_{1}}^{\infty} \min \left\{\left(\mu+\left\|a_{-}\right\|_{\infty}\right)^{3 / 2},\left(s+\left\|a_{-}\right\|_{\infty}\right)^{3 / 2}\right\} \frac{d s}{s^{2}} .
\end{aligned}
$$

The integral is easily seen to be bounded by a universal constant times

$$
\mu^{1 / 2}+E_{1}^{-1}\left\|a_{-}\right\|_{\infty}^{3 / 2}
$$

This proves the claimed bound.

Lemma 5.4 There are constants $T_{*}<\infty, \rho>0$ and $C<\infty$ such that for all $x \in \Omega, \lambda>0$ with $d \lambda \geq T_{*}$, and all $v \in T_{x, \lambda}^{\perp}$ and all $\mu \geq 1$ the function

$$
v_{>}:=\mathbb{1}(-\Delta+a>\mu) v
$$


satisfies

$$
\int_{\Omega}\left(\left|\nabla v_{>}\right|^{2}+a v_{>}^{2}-15 U_{x, \lambda}^{4} v_{>}^{2}\right) d y \geq \rho \int_{\Omega}\left|\nabla v_{>}\right|^{2} d y-C \mu^{1 / 2} \lambda^{-1}\|\nabla v\|^{2} .
$$

Proof Step 1 We construct an orthonormal basis in $T_{x, \lambda}=\operatorname{Span}\left\{\phi_{1}, \ldots, \phi_{5}\right\}$, where

$$
\phi_{1}=P U_{x, \lambda}, \quad \phi_{2}=\partial_{\lambda} P U_{x, \lambda}, \quad \phi_{j}=\partial_{x_{j-2}} P U_{x, \lambda}, \quad j=3,4,5 .
$$

From [24, Appendix B] we know that, as $\lambda \rightarrow \infty$,

$$
\left\|\nabla \phi_{1}\right\| \sim 1, \quad\left\|\nabla \phi_{2}\right\| \sim \lambda^{-1}, \quad\left\|\nabla \phi_{j}\right\| \sim \lambda, \quad j=3,4,5,
$$

uniformly in $x$ with $\lambda d \geq T_{*}$, where $T_{*}$ is any fixed constant. Here $\sim$ means that the quotient of both quantities is bounded from above and away from zero. Let

$$
\tilde{\phi}_{j}:=\frac{\phi_{j}}{\left\|\nabla \phi_{j}\right\|}, \quad j=1, \ldots, 5,
$$

and

$$
G_{j, k}:=\int_{\Omega} \nabla \tilde{\phi}_{j} \cdot \nabla \tilde{\phi}_{k} d y, \quad j, k=1, \ldots, 5 .
$$

By [24, Appendix B] and (5.10),

$$
G_{j, k}:=\mathcal{O}\left(\lambda^{-1}\right) \text { for all } j \neq k \text { and } G_{j, j}=1 \text { for all } j .
$$

Hence, if $\lambda$ is large enough, which follows from $d \lambda \geq T_{*}$ with sufficiently large $T_{*}$ since $\Omega$ is bounded, then $G$ is invertible and

$$
\left(G^{-1 / 2}\right)_{j, k}=\delta_{j, k}+\mathcal{O}\left(\lambda^{-1}\right) .
$$

Hence, by the Gram-Schmidt procedure,

$$
\psi_{j}:=\sum_{k}\left(G^{-1 / 2}\right)_{j, k} \tilde{\phi}_{k} \quad j=1, \ldots, 5,
$$

is an $H_{0}^{1}(\Omega)$-orthonormal basis of $T_{x, \lambda}$.

Step 2 We decompose

$$
v_{>}=v_{\|}+v_{\perp} \text { with } v_{\|} \in T_{x, \lambda} \text { and } v_{\perp} \in T_{x, \lambda}^{\perp}
$$

and claim that

$$
\left\|\nabla v_{\|}\right\|=\mathcal{O}\left(\lambda^{-1 / 2} \mu^{1 / 4}\|\nabla v\|\right) .
$$

Since the $\psi_{j}$ are an orthonormal basis of $T_{x, \lambda}$, we have

$$
v_{\|}=\sum_{j=1}^{5} m_{j} \psi_{j} \quad \text { with } \quad m_{j}:=\int_{\Omega} \nabla \psi_{j} \cdot \nabla v_{>} d y .
$$

Since

$$
\int_{\Omega}\left|\nabla v_{\|}\right|^{2} d y=\sum_{j} m_{j}^{2}
$$

the claim (5.16) follows from

$$
m_{j}=\mathcal{O}\left(\lambda^{-1 / 2} \mu^{1 / 4}\|\nabla v\|\right) \text { for all } j=1, \ldots, 5 .
$$


In order to prove the latter, we introduce

$$
\ell_{j}:=\int_{\Omega} \nabla \tilde{\phi}_{j} \cdot \nabla v_{>} d y
$$

so that, by (5.14),

$$
m_{j}=\sum_{k}\left(G^{-1 / 2}\right)_{j, k} l_{k}
$$

Therefore, in view of (5.13), the claim (5.17) follows from

$$
\ell_{j}=\mathcal{O}\left(\lambda^{-1 / 2} \mu^{1 / 4}\|\nabla v\|\right) \text { for all } j=1, \ldots, 5 .
$$

To prove (5.18), we use the fact that $v \in T_{x, \lambda}^{\perp}$ to find

$$
\ell_{j}=-\int_{\Omega} \nabla \tilde{\phi}_{j} \cdot \nabla v_{<} d y=\int_{\Omega} v_{<} \Delta \tilde{\phi}_{j} d y
$$

Thus,

$$
\left|\ell_{j}\right| \leq\left\|v_{<}\right\|_{\infty}\left\|\Delta \tilde{\phi}_{j}\right\|_{1}
$$

According to (5.7) we have $\left\|v_{<}\right\|_{\infty} \lesssim \mu^{1 / 4}\|\nabla v\|$. Thus, in order to complete the proof of (5.18) we need to show that $\left\|\Delta \tilde{\phi}_{j}\right\|_{1}=\mathcal{O}\left(\lambda^{-1 / 2}\right)$ for $j=1, \ldots, 5$. We have

$$
\begin{aligned}
& -\Delta \tilde{\phi}_{1}=\left\|\nabla \phi_{1}\right\|^{-1} 3 U_{x, \lambda}^{5}, \quad-\Delta \tilde{\phi}_{2}=\left\|\nabla \phi_{2}\right\|^{-1} 15 U_{x, \lambda}^{4} \partial_{\lambda} U_{x, \lambda}, \\
& -\Delta \tilde{\phi}_{j}=\left\|\nabla \phi_{j}\right\|^{-1} 15 U_{x, \lambda}^{4} \partial_{j} U_{x, \lambda} \quad \text { for } j=3,4,5 .
\end{aligned}
$$

Thus, the claimed bound on $\left\|\Delta \tilde{\phi}_{j}\right\|_{1}$ follows from (5.10) and straightforward bounds on $\left\|U_{x, \lambda}\right\|_{5},\left\|\partial_{\lambda} U_{x, \lambda}\right\|_{5}$ and $\left\|\partial_{j} U_{x, \lambda}\right\|_{5}$. This completes the proof of (5.18) and therefore of (5.16).

Step 3 By the orthogonal decomposition (5.15) we have

$$
\int_{\Omega}\left|\nabla v_{>}\right|^{2} d y=\int_{\Omega}\left|\nabla v_{\|}\right|^{2} d y+\int_{\Omega}\left|\nabla v_{\perp}\right|^{2} d y .
$$

Moreover, we bound, with a parameter $\delta>0$ to be determined,

$$
\int_{\Omega} U_{x, \lambda}^{4} v_{>}^{2} d y \leq\left(1+\delta^{-1}\right) \int_{\Omega} U_{x, \lambda}^{4} v_{\|}^{2} d y+(1+\delta) \int_{\Omega} U_{x, \lambda}^{4} v_{\perp}^{2} d y
$$

and

$$
\int_{\Omega} a v_{>}^{2} d y \geq-\left(1+\delta^{-1}\right) \int_{\Omega}|a| v_{\|}^{2} d y+\int_{\Omega} a v_{\perp}^{2} d y-\delta \int_{\Omega}|a| v_{\perp}^{2} d y .
$$

Thus,

$$
\begin{aligned}
\int_{\Omega}\left(\left|\nabla v_{>}\right|^{2}+a v_{>}^{2}-15 U_{x, \lambda} v_{>}^{2}\right) d y \geq & \int_{\Omega}\left(\left|\nabla v_{\perp}\right|^{2}+a v_{\perp}^{2}-15 U_{x, \lambda} v_{\perp}^{2}\right) d y \\
& -\delta \int_{\Omega}\left(|a|+15 U_{x, \lambda}^{4}\right) v_{\perp}^{2} d y \\
& +\int_{\Omega}\left|\nabla v_{\|}\right|^{2} d y-\left(1+\delta^{-1}\right) \int_{\Omega}\left(|a|+15 U_{x, \lambda}^{4}\right) v_{\|}^{2} d y .
\end{aligned}
$$


Clearly,

$$
\int_{\Omega}\left(|a|+15 U_{x, \lambda}^{4}\right) z^{2} d y \leq\left(\|a\|_{3 / 2}+15\left\|U_{x, \lambda}\right\|_{6}^{4}\right)\|z\|_{6}^{2} \lesssim\|\nabla z\|^{2} \forall z \in H_{0}^{1}(\Omega) .
$$

Since $v_{\perp} \in T_{x, \lambda}^{\perp}$, Lemma 4.3 and (5.20) imply that, after increasing $T_{*}$ if necessary, there are $\delta>0$ and $c>0$ such that

$$
\int_{\Omega}\left(\left|\nabla v_{\perp}\right|^{2}+a v_{\perp}^{2}-15 U_{x, \lambda} v_{\perp}^{2}\right) d y-\delta \int_{\Omega}\left(|a|+15 U_{x, \lambda}^{4}\right) v_{\perp}^{2} d y \geq c \int_{\Omega}\left|\nabla v_{\perp}\right|^{2} d y .
$$

On the other hand, by (5.20) and (5.16),

$$
\int_{\Omega}\left(|a|+15 U_{x, \lambda}^{4}\right) v_{\|}^{2} d y \lesssim \int_{\Omega}\left|\nabla v_{\|}\right|^{2} d y=\mathcal{O}\left(\lambda^{-1} \mu^{1 / 2}\|\nabla v\|^{2}\right)
$$

This completes the proof of Lemma 5.4.

\subsection{Completing the square}

The following lemma gives a lower bound on the term in (5.4) which involves $w$. As explained above, this is the crucial step in the proof of Proposition 5.1.

Lemma 5.5 For some constant $c>0$,

$$
\begin{aligned}
& \int_{\Omega}\left(|\nabla w|^{2}+a w^{2}+2 \lambda^{-1 / 2} a G_{0}(x, y) w-15 U_{x, \lambda}^{4} w^{2}\right) d y \\
& \geq-\lambda^{-1}(4 \pi)^{-1} \iint_{\Omega \times \Omega} G_{0}(x, y) a(y) G_{a}\left(y, y^{\prime}\right) a\left(y^{\prime}\right) G_{0}\left(y^{\prime}, x\right) d y d y^{\prime} \\
& \quad+c\left\|(-\Delta+a)^{1 / 2} w+(-\Delta+a)^{-1 / 2} \lambda^{-1 / 2} a G_{0}(x, \cdot)\right\|^{2}+\mathcal{O}\left(\lambda^{-3 / 2}\right) .
\end{aligned}
$$

Proof For a parameter $\mu \geq 1$ to be specified later we decompose $w=w_{>}+w_{<}$with

$$
w_{>}=\mathbb{1}(-\Delta+a>\mu) w, \quad w_{<}=\mathbb{1}(-\Delta+a \leq \mu) w .
$$

Then

$$
\int_{\Omega}\left(|\nabla w|^{2}+a w^{2}\right) d y=\int_{\Omega}\left(\left|\nabla w_{>}\right|^{2}+a w_{>}^{2}\right) d y+\int_{\Omega}\left(\left|\nabla w_{<}\right|^{2}+a w_{<}^{2}\right) d y
$$

and therefore, for any $\delta>0$,

$$
\int_{\Omega}\left(|\nabla w|^{2}+a w^{2}+2 \lambda^{-1 / 2} a G_{0}(x, y) w-15 U_{x, \lambda}^{4} w^{2}\right) d y \geq I_{<}+I_{>}+R_{<}(\delta)+R_{>}(\delta),
$$

where

$$
\begin{aligned}
I_{<} & :=\int_{\Omega}\left(\left|\nabla w_{<}\right|^{2}+a w_{<}^{2}+2 \lambda^{-1 / 2} a G_{0}(x, y) w_{<}\right) d y, \\
I_{>} & :=\int_{\Omega}\left(\left|\nabla w_{>}\right|^{2}+a w_{>}^{2}-15 U_{x, \lambda}^{4} w_{>}^{2}\right) d y, \\
R_{<}(\delta) & :=-15\left(1+\delta^{-1}\right) \int_{\Omega} U_{x, \lambda}^{4} w_{<}^{2} d y, \\
R_{>}(\delta) & :=-15 \delta \int_{\Omega} U_{x, \lambda}^{4} w_{>}^{2} d y+2 \lambda^{-1 / 2} \int_{\Omega} a G_{0}(x, y) w_{>} d y .
\end{aligned}
$$


By completing the square we find

$$
\begin{aligned}
I_{<}= & -\lambda^{-1}(4 \pi)^{-1} \iint_{\Omega \times \Omega} G_{0}(x, y) a(y) G_{a}\left(y, y^{\prime}\right) a\left(y^{\prime}\right) G_{0}\left(y^{\prime}, x\right) d y d y^{\prime} \\
& +\left\|(-\Delta+a)^{1 / 2} w_{<}+(-\Delta+a)^{-1 / 2} \lambda^{-1 / 2} a G_{0}(x, \cdot)\right\|^{2}
\end{aligned}
$$

and with $0 \leq c \leq 1$ to be determined we estimate

$$
\begin{aligned}
I_{<} \geq & -\lambda^{-1}(4 \pi)^{-1} \iint_{\Omega \times \Omega} G_{0}(x, y) a(y) G_{a}\left(y, y^{\prime}\right) a\left(y^{\prime}\right) G_{0}\left(y^{\prime}, x\right) d y d y^{\prime} \\
& +c\left\|(-\Delta+a)^{1 / 2} w_{<}+(-\Delta+a)^{-1 / 2} \lambda^{-1 / 2} a G_{0}(x, \cdot)\right\|^{2} \\
= & -\lambda^{-1}(4 \pi)^{-1} \iint_{\Omega \times \Omega} G_{0}(x, y) a(y) G_{a}\left(y, y^{\prime}\right) a\left(y^{\prime}\right) G_{0}\left(y^{\prime}, x\right) d y d y^{\prime} \\
& +c\left\|(-\Delta+a)^{1 / 2} w+(-\Delta+a)^{-1 / 2} \lambda^{-1 / 2} a G_{0}(x, \cdot)\right\|^{2} \\
& -c\left\|(-\Delta+a)^{1 / 2} w_{>}\right\|^{2}-2 c \lambda^{-1 / 2} \int_{\Omega} a G_{0}(x, y) w_{>} d y .
\end{aligned}
$$

According to Lemma 5.4 there are $\rho>0$ and $C<\infty$ such that for all sufficiently small $\epsilon>0$,

$$
I_{>} \geq \rho \int_{\Omega}\left|\nabla w_{>}\right|^{2} d y-C \mu^{1 / 2} \lambda^{-1}\|\nabla w\|^{2} .
$$

Since $a \in L^{\infty}(\Omega)$, we have

$$
\left\|(-\Delta+a)^{1 / 2} z\right\|^{2} \leq C^{\prime}\|\nabla z\|^{2} \quad \forall z \in H_{0}^{1}(\Omega) .
$$

We apply this with $u=w_{>}$and infer that

$$
\begin{aligned}
I_{<} & +I_{>}(\delta)+R_{<}(\delta)+R_{>} \\
\geq & -\lambda^{-1}(4 \pi)^{-1} \iint_{\Omega \times \Omega} G_{0}(x, y) a(y) G_{a}\left(y, y^{\prime}\right) a\left(y^{\prime}\right) G_{0}\left(y^{\prime}, x\right) d y d y^{\prime} \\
& +c\left\|(-\Delta+a)^{1 / 2} w+(-\Delta+a)^{-1 / 2} \frac{\alpha}{\sqrt{\lambda}} a G_{0}(x, \cdot)\right\|^{2}+R_{1}(\delta)+R_{2}(\delta),
\end{aligned}
$$

where

$$
\begin{aligned}
R_{1}(\delta) & =\rho\left\|\nabla w_{>}\right\|^{2}-c C^{\prime}\left\|\nabla w_{>}\right\|^{2}-15 \delta \int_{\Omega} U_{x, \lambda}^{4} w_{>}^{2} d y \\
R_{2}(\delta) & =-C \mu^{1 / 2} \lambda^{-1}\|\nabla w\|^{2}+2(1-c) \lambda^{-1 / 2} \int_{\Omega} a G_{0}(x, y) w_{>} d y \\
& -15\left(1+\delta^{-1}\right) \int_{\Omega} U_{x, \lambda}^{4} w_{<}^{2} d y
\end{aligned}
$$

We now choose $c=\min \left\{1, \rho /\left(2 C^{\prime}\right)\right\}$. Moreover, by (5.20) we can choose a $\delta>0$, independent of $\epsilon$ and $\mu$ such that

$$
R_{1}(\delta) \geq 0
$$

From now on, we fix this value of $\delta$. 
It remains to show that $R_{2}(\delta)$ is $\mathcal{O}\left(\lambda^{-3 / 2}\right)$ for an appropriate choice of $\mu$. By (4.1) and (5.25) and by the orthogonality (5.22) we have

$$
\mathcal{O}\left(\lambda^{-1}\right)=\int_{\Omega}|\nabla w|^{2} d y \gtrsim \int_{\Omega}\left(|\nabla w|^{2}+a w^{2}\right) d y \geq \int_{\Omega}\left(\left|\nabla w_{>}\right|^{2}+a w_{>}^{2}\right) d y \geq \mu\left\|w_{>}\right\|^{2} .
$$

Thus, since $a \in L^{\infty}(\Omega)$ and since $G_{0}(x, \cdot)$ is uniformly bounded in $L^{2}(\Omega)$, we have

$$
\left|\int_{\Omega} a G_{0}(x, y) w_{>} d y\right| \lesssim\left\|w_{>}\right\| \lesssim \mu^{-1 / 2} \lambda^{-1 / 2} .
$$

Moreover, by Lemma 5.3,

$$
\int_{\Omega} U_{x, \lambda}^{4} w_{<}^{2} d y \leq\left\|w_{<}\right\|_{\infty}^{2} \int_{\Omega} U_{x, \lambda}^{4} d y \lesssim \mu^{1 / 2}\|\nabla w\|^{2} \int_{\mathbb{R}^{3}} U_{x, \lambda}^{4} d y \lesssim \mu^{1 / 2} \lambda^{-2} .
$$

Thus,

$$
R_{2}(\delta) \gtrsim-\left(\mu^{1 / 2} \lambda^{-2}+\mu^{-1 / 2} \lambda^{-1}\right) .
$$

With the choice $\mu=\lambda$ the right side becomes $\mathcal{O}\left(\lambda^{-3 / 2}\right)$, as claimed.

Now we prove the main result of this section.

Proof of Proposition 5.1 Inserting (5.21) into (5.4) gives

$$
\begin{aligned}
\mathcal{S}_{a+\epsilon V}\left[u_{\varepsilon}\right] \geq & S+4 \pi \lambda^{-1}(S / 3)^{-1 / 2} \phi_{a}(x) \\
& +(S / 3)^{-1 / 2} c\left\|(-\Delta+a)^{1 / 2} w+(-\Delta+a)^{-1 / 2} \lambda^{-1 / 2} a G_{0}(x, \cdot)\right\|^{2}+o\left(\lambda^{-1}\right) .
\end{aligned}
$$

We subtract $S(a+\epsilon V)$ from both sides, multiply by $\lambda$ and take the limsup as $\epsilon \rightarrow 0+$. Using the second relation in (4.3) we obtain

$$
\begin{aligned}
0 \geq & \limsup _{\epsilon \rightarrow 0}\left(\lambda(S-S(a+\epsilon V))+4 \pi(S / 3)^{-1 / 2} \phi_{a}(x)\right. \\
& \left.+(S / 3)^{-1 / 2} c \lambda\left\|(-\Delta+a)^{1 / 2} w+(-\Delta+a)^{-1 / 2} \lambda^{-1 / 2} a G_{0}(x, \cdot)\right\|^{2}\right) .
\end{aligned}
$$

Since the three terms in the limsup are all non-negative (which for $\phi_{a}$ follows from Corollary 2.2), we deduce that

$$
\lambda(S-S(a+\epsilon V))=o(1), \quad \phi_{a}(x)=o(1)
$$

and

$$
\left\|(-\Delta+a)^{1 / 2} w+(-\Delta+a)^{-1 / 2} \lambda^{-1 / 2} a G_{0}(x, \cdot)\right\|^{2}=o\left(\lambda^{-1}\right) .
$$

Since $-\Delta+a$ is coercive, the last bound implies

$$
\left\|\nabla\left(w+(-\Delta+a)^{-1} \lambda^{-1 / 2} a G_{0}(x, \cdot)\right)\right\|^{2}=o\left(\lambda^{-1}\right) .
$$

By the resolvent identity,

$$
(-\Delta+a)^{-1} a G_{0}(x, \cdot)=G_{0}(x, \cdot)-G_{a}(x, \cdot)=H_{a}(x, \cdot)-H_{0}(x, \cdot),
$$

and therefore, setting $q:=w+\lambda^{-1 / 2}\left(H_{a}(x, \cdot)-H_{0}(x, \cdot)\right)$, the previous bound can be rewritten as $\|\nabla q\|^{2}=o\left(\lambda^{-1}\right)$. This completes the proof of the proposition. 


\section{A refined decomposition of almost minimizers}

From Proposition 5.1 we infer that any sequence $\left(u_{\varepsilon}\right)$ satisfying (1.10) can be decomposed as

$$
u_{\epsilon}=\alpha\left(\psi_{x, \lambda}+q\right)
$$

where

$$
\psi_{x, \lambda}=P U_{x, \lambda}-\lambda^{-1 / 2}\left(H_{a}(x, \cdot)-H_{0}(x, \cdot)\right)
$$

is as in the proof of the upper bound, see (2.1), and where

$$
\|\nabla q\|=o\left(\lambda^{-1 / 2}\right) .
$$

Thus, expanding $\mathcal{S}_{a+\epsilon V}\left[u_{\epsilon}\right]$ leads to an expression that coincides with the upper bound in Corollary 2.2 up to additional terms involving $q$. Using coercivity we will be able to show that the contribution from

$$
r:=\Pi_{x, \lambda}^{\perp} q,
$$

the orthogonal projection of $q$ onto $T_{x, \lambda}^{\perp}$ in $H_{0}^{1}(\Omega)$, is negligible; see Lemma 6.6 below. The main focus in this section is on

$$
\Pi_{x, \lambda} q=\Pi_{x, \lambda}\left(w+\lambda^{-1 / 2}\left(H_{a}(x, \cdot)-H_{0}(x, \cdot)\right)\right)=\lambda^{-1 / 2} \Pi_{x, \lambda}\left(H_{a}(x, \cdot)-H_{0}(x, \cdot)\right),
$$

where the last identity follows from $w \in T_{x, \lambda}^{\perp}$. In Lemma 6.3 we will prove that the contribution from $\Pi_{x, \lambda} q$ is negligible. This is not obvious and, in fact, somewhat surprising since $\Pi_{x, \lambda} q$ is of order $\lambda^{-1}$ and not smaller.

\subsection{Preliminary estimates}

Let us write

$$
\Pi_{x, \lambda} q=\beta \lambda^{-1} P U_{x, \lambda}+\gamma \partial_{\lambda} P U_{x, \lambda}+\sum_{j=1}^{3} \delta_{j} \lambda^{-3} \partial_{x_{j}} P U_{x, \lambda} .
$$

Since $P U_{x, \lambda}, \partial_{\lambda} P U_{x, \lambda}$ and $\partial_{x_{j}} P U_{x, \lambda}, j=1,2,3$, are linearly independent for sufficiently large $\lambda$, the numbers $\beta, \gamma$ and $\delta_{j}, j=1,2,3$, (depending on $\epsilon$, of course) are uniquely determined. The choice of the different powers of $\lambda$ multiplying these coefficients is motivated by the following lemma.

Lemma 6.1 As $\epsilon \rightarrow 0$, we have

$$
\beta, \gamma, \delta_{j}=\mathcal{O}(1) \text {. }
$$

Proof We recall that the functions $\tilde{\phi}_{j}, j=1, \ldots, 5$, were introduced in (5.11). Let

$$
a_{j}:=\int_{\Omega} \nabla \tilde{\phi}_{j} \cdot \nabla q d y, \quad j=1, \ldots, 5 .
$$

Step 1 We shall show that

$$
a_{1}, a_{2}=\mathcal{O}\left(\lambda^{-1}\right), \quad a_{3}, a_{4}, a_{5}=\mathcal{O}\left(\lambda^{-2}\right) .
$$


Since $-\lambda^{-1 / 2}\left(H_{a}(x, \cdot)-H_{0}(x, \cdot)\right)+q=w \in T_{x, \lambda}^{\perp}$, we have

$$
\begin{aligned}
a_{j} & =\lambda^{-1 / 2} \int_{\Omega} \nabla \tilde{\phi}_{j} \cdot \nabla_{y}\left(H_{a}(x, y)-H_{0}(x, y)\right) d y \\
& =-\lambda^{-1 / 2} \int_{\Omega}\left(\Delta \tilde{\phi}_{j}\right)\left(H_{a}(x, y)-H_{0}(x, y)\right) d y .
\end{aligned}
$$

Formulas for the Laplacians $\Delta \tilde{\phi}_{j}$ are given in (5.19) and the quantities $\left\|\nabla \phi_{j}\right\|$ appearing there were estimated in (5.10). For $a_{1}$, the integral $\int_{\Omega} U_{x, \lambda}^{5}\left(H_{a}(x, y)-H_{0}(x, y)\right) d y$ is $\mathcal{O}\left(\lambda^{-1 / 2}\right)$ according to Lemma 2.5, which proves the claim in (6.1). To bound $a_{j}$ for $j=2, \ldots, 5$ we compute

$$
\begin{aligned}
\partial_{\lambda} U_{x, \lambda}(y) & =\frac{\lambda^{-1 / 2}}{2} \frac{1-\lambda^{2}|y-x|^{2}}{\left(1+\lambda^{2}|y-x|^{2}\right)^{3 / 2}}, \\
\partial_{x_{i}} U_{x, \lambda}(y) & =\lambda^{5 / 2} \frac{y_{i}-x_{i}}{\left(1+\lambda^{2}|y-x|^{2}\right)^{3 / 2}}, \quad i=1,2,3 .
\end{aligned}
$$

This expression and straightforward bounds lead to the claim for $a_{2}$ in (6.1).

To prove (6.1) for $a_{j}$ with $j=3,4,5$ we need to bound

$$
\int_{\Omega}\left(H_{a}(x, y)-H_{0}(x, y)\right) U_{x, \lambda}^{4} \partial_{x_{j}} U_{x, \lambda} d y .
$$

From Step 1 in the proof of Lemma 2.5, recalling (4.2), we infer that there are $\rho>0$ and $C>0$, both independent of $\epsilon$, such that

$$
\left|H_{a}(x, y)-H_{0}(x, y)-H_{a}(x, x)+H_{0}(x, x)\right| \lesssim|y-x| \text { for all } y \in B_{\rho}(x) .
$$

Since the function $U_{x, \lambda}^{4} \partial_{x_{j}} U_{x, \lambda}$ is odd, we have

$$
\int_{B_{\rho}(x)}\left(H_{a}(x, x)-H_{0}(x, x)\right) U_{x, \lambda}^{4} \partial_{x_{j}} U_{x, \lambda} d y=0 .
$$

On the other hand, using the above expression for $\partial_{x_{j}} U_{x, \lambda}$ we find

$$
\int_{\Omega} \min \{|y-x|, \rho\}\left|U_{x, \lambda}^{4} \partial_{x_{j}} U_{x, \lambda}\right| d y=\mathcal{O}\left(\lambda^{-1 / 2}\right) .
$$

This proves (6.1) for $j=3,4,5$.

Step 2 Let us deduce the statement of the lemma. We have

$$
\Pi_{x, \lambda} q=\sum_{j=1}^{5} \tilde{a}_{j} \tilde{\phi}_{j}
$$

with

$$
\begin{array}{r}
\tilde{a}_{1}:=\beta \lambda^{-1}\left\|\nabla P U_{x, \lambda}\right\|, \quad \tilde{a}_{2}:=\gamma\left\|\nabla \partial_{\lambda} P U_{x, \lambda}\right\|, \quad \tilde{a}_{j} \\
:=\delta_{j} \lambda^{-3}\left\|\nabla \partial_{x_{j-2}} P U_{x, \lambda}\right\|, \quad j=3,4,5 .
\end{array}
$$

In view of (5.10), the assertion of the lemma is equivalent to

$$
\tilde{a}_{1}, \tilde{a}_{2}=\mathcal{O}\left(\lambda^{-1}\right), \quad \tilde{a}_{j}=\mathcal{O}\left(\lambda^{-2}\right), \quad j=3,4,5 .
$$


With respect to the orthonormal system $\psi_{j}, j=1, \ldots, 5$, from (5.14) we have

$$
\Pi_{x, \lambda} q=\sum_{j=1}^{5}\left(\nabla \psi_{j}, \nabla q\right) \psi_{j}
$$

Using (5.14) twice to express $\psi_{j}$ in terms of $\tilde{\phi}_{k}$ 's we obtain

$$
\Pi_{x, \lambda} q=\sum_{k=1}^{5} \sum_{\ell=1}^{5}\left(G^{-1}\right)_{k, \ell}\left(\nabla \tilde{\phi}_{\ell}, \nabla q\right) \tilde{\phi}_{k}=\sum_{k=1}^{5} \sum_{\ell=1}^{5}\left(G^{-1}\right)_{k, \ell} a_{\ell} \tilde{\phi}_{k} .
$$

Thus,

$$
\tilde{a}_{k}=\sum_{\ell=1}^{5}\left(G^{-1}\right)_{k, \ell} a_{\ell}, \quad k=1, \ldots, 5 .
$$

Similarly as in (5.13) one finds

$$
\left(G^{-1}\right)_{j, k}=\delta_{j, k}+\mathcal{O}\left(\lambda^{-1}\right),
$$

and then (6.2) follows from (6.1). This completes the proof of the lemma.

Remark 6.2 The same method of proof shows that there are non-zero numbers $\beta_{0}, \gamma_{0}, \delta_{0, j}$ such that

$$
\beta \rightarrow \beta_{0}, \quad \gamma \rightarrow \gamma_{0}, \quad \delta_{0, j} \rightarrow \delta_{0}
$$

as $\epsilon \rightarrow 0$. Indeed, proceeding as in Step 1 above one can show that $\lambda a_{k}$ for $k=1,2$ and $\lambda^{2} a_{k}$ for $k=3,4,5$ have a non-zero limit as $\epsilon \rightarrow 0$. As in Step 2 above, this implies that $\lambda \tilde{a}_{k}$ for $k=1,2$ have a non-zero limit as $\epsilon \rightarrow 0$. In order to compute the limits of $\lambda \tilde{a}_{k}$ for $k=3,4,5$ one needs to use, in addition, the fact that $\left(G^{-1}\right)_{k, \ell}=\delta_{k, \ell}+\mathcal{O}\left(\lambda^{-2}\right)$ for $k=3,4,5$. Indeed, by a Neumann series for $G=1-(1-G)$ one finds

$$
\left(G^{-1}\right)_{k, \ell}=(2-G)_{k, \ell}+\mathcal{O}\left(\lambda^{-2}\right)=2 \delta_{k, \ell}-\int_{\Omega} \nabla \tilde{\phi}_{k} \cdot \nabla \tilde{\phi}_{\ell} d y+\mathcal{O}\left(\lambda^{-2}\right),
$$

and then one can use bounds from [24, Appendix B] for the integral on the right side.

\subsection{A third expansion}

In this subsection, we shall prove the following lemma.

Lemma 6.3 As $\epsilon \rightarrow 0$,

$$
\mathcal{S}_{a+\epsilon V}\left[u_{\epsilon}\right]=\mathcal{S}_{a+\epsilon V}\left[\psi_{x, \lambda}\right]+(S / 3)^{-1 / 2}\left(\mathcal{E}_{0}[r]-\frac{N_{0}}{3 D_{0}} \mathcal{I}[r]\right)+o\left(\lambda^{-2}\right)+o\left(\epsilon \lambda^{-1}\right)
$$

with

$$
N_{0}:=\int_{\Omega}\left(\left|\nabla \psi_{x, \lambda}\right|^{2}+(a+\epsilon V) \psi_{x, \lambda}^{2}\right) d y, \quad D_{0}:=\int_{\Omega} \psi_{x, \lambda}^{6} d y
$$

and

$$
\mathcal{I}[r]:=-30 \lambda^{-1 / 2} \int_{\Omega} U_{x, \lambda}^{4} H_{a}(x, y) r d y+15 \int_{\Omega} U_{x, \lambda}^{4} r^{2} d y+20 \int_{\Omega} U_{x, \lambda}^{3} r^{3} d y .
$$


We emphasize that the coefficients $\beta, \gamma$ and $\delta_{j}$ enter only into the remainders $o\left(\lambda^{-2}\right)+$ $o\left(\epsilon \lambda^{-1}\right)$. This is somewhat surprising since $\beta$ enters to orders $\lambda^{-1}$ and $\lambda^{-2}$ and $\gamma$ enters to order $\lambda^{-2}$ in the expansion of the numerator and the denominator.

In the following, it will be convenient to abbreviate

$$
g:=\beta \lambda^{-1} P U_{x, \lambda}+\gamma \partial_{\lambda} P U_{x, \lambda}, \quad h:=\sum_{j=1}^{3} \delta_{j} \lambda^{-3} \partial_{x_{j}} P U_{\lambda, x},
$$

so that

$$
u=\alpha\left(\psi_{x, \lambda}+g+h+r\right)
$$

We record the bounds

$$
\|\nabla g\|=\mathcal{O}\left(\lambda^{-1}\right), \quad\|\nabla h\|=\mathcal{O}\left(\lambda^{-2}\right), \quad\|\nabla r\|=o\left(\lambda^{-1 / 2}\right) .
$$

Indeed, the bounds on $g$ and $h$ follow from Lemma 6.1 together with (5.10) and that for $r$ follows from Proposition 5.1 since, by orthogonality, $\|\nabla r\| \leq\|\nabla q\|$.

We will also use the fact that

$$
\|\Delta h\|_{1}=\mathcal{O}\left(\lambda^{-5 / 2}\right) .
$$

This follows from Lemma 6.1 together with (5.19) and the same bounds that led to (5.18).

We will obtain Lemma 6.3 from separate expansions of the numerator and the denominator, which we state in the following two lemmas.

Expanding the numerator We abbreviate

$$
\mathcal{E}_{\epsilon}[v]:=\int_{\Omega}\left(|\nabla v|^{2}+(a+\epsilon V) v^{2}\right) d y
$$

and write $\mathcal{E}_{\epsilon}\left[v_{1}, v_{2}\right]$ for the associated bilinear form. Recall that $N_{0}$ was defined in (6.4). We shall show

Lemma 6.4 As $\epsilon \rightarrow 0$,

$$
\alpha^{-2} \mathcal{E}_{\epsilon}\left[u_{\epsilon}\right]=N_{0}+N_{1}+\mathcal{E}_{0}[r]+o\left(\lambda^{-2}\right)+o\left(\epsilon \lambda^{-1}\right),
$$

where

$$
N_{1}:=\int_{\Omega}|\nabla g|^{2} d y+2 \mathcal{E}_{0}\left[\psi_{x, \lambda}, g\right]
$$

Proof Step 1 We show that the contribution from $h$ to $\alpha^{-2} \mathcal{E}_{\epsilon}\left[u_{\epsilon}\right]$ is negligible, that is,

$$
\alpha^{-2} \mathcal{E}_{\epsilon}\left[u_{\epsilon}\right]=\mathcal{E}_{\epsilon}\left[\psi_{x, \lambda}+g+r\right]+o\left(\lambda^{-5 / 2}\right) .
$$

Indeed,

$$
\alpha^{-2} \mathcal{E}_{\epsilon}\left[u_{\epsilon}\right]=\mathcal{E}_{\epsilon}\left[\psi_{x, \lambda}+g+r\right]+2 \mathcal{E}_{\epsilon}\left[\psi_{x, \lambda}+g+r, h\right]+\mathcal{E}_{\epsilon}[h] .
$$

Since $\mathcal{E}_{\epsilon}\left[v_{1}, v_{2}\right] \lesssim\left\|\nabla v_{1}\right\|\left\|\nabla v_{2}\right\|$ for all $v_{1}, v_{2} \in H_{0}^{1}(\Omega)$, we immediately conclude from (6.6) that

$$
\mathcal{E}_{\epsilon}[h]=\mathcal{O}\left(\lambda^{-4}\right) \quad \mathcal{E}_{\epsilon}[g+r, h]=o\left(\lambda^{-5 / 2}\right)
$$


Next, using (6.7), (2.6) and (2.7),

$$
\begin{aligned}
\int_{\Omega} \nabla \psi_{x, \lambda} \cdot \nabla h d y & =\int_{\Omega} \nabla P U_{x, \lambda} \cdot \nabla h d y+\mathcal{O}\left(\lambda^{-1 / 2}\left\|H_{a}(x, \cdot)-H_{0}(x, \cdot)\right\|_{\infty}\|\Delta h\|_{1}\right) \\
& =\int_{\Omega} \nabla P U_{x, \lambda} \cdot \nabla h d y+\mathcal{O}\left(\lambda^{-3}\right) .
\end{aligned}
$$

Moreover, by (5.12) and (5.10),

$$
\int_{\Omega} \nabla P U_{x, \lambda} \cdot \nabla h d y=\sum_{j=1}^{3} \delta_{j} \lambda^{-3} \int_{\Omega} \nabla P U_{x, \lambda} \cdot \nabla \partial_{x_{j}} P U_{x, \lambda} d y=\mathcal{O}\left(\lambda^{-3}\right) .
$$

Finally, by (A.8) and (6.6),

$$
\left|\int_{\Omega}(a+\epsilon V) \psi_{x, \lambda} h d y\right| \leq\|a+\epsilon V\|_{\infty}\left\|\psi_{x, \lambda}\right\|_{6 / 5}\|h\|_{6}=\mathcal{O}\left(\lambda^{-5 / 2}\right) .
$$

This proves (6.8).

Step 2 We now extract the relevant contribution from $g$ and show

$$
\mathcal{E}_{\epsilon}\left[\psi_{x, \lambda}+g+r\right]=\mathcal{E}_{\epsilon}\left[\psi_{x, \lambda}+r\right]+2 \mathcal{E}_{0}\left[\psi_{x, \lambda}, g\right]+\int_{\Omega}|\nabla g|^{2} d y+o\left(\lambda^{-2}\right)
$$

Indeed,

$$
\mathcal{E}_{\epsilon}\left[\psi_{x, \lambda}+g+r\right]=\mathcal{E}_{\epsilon}\left[\psi_{x, \lambda}+r\right]+2 \mathcal{E}_{\epsilon}\left[\psi_{x, \lambda}+r, g\right]+\mathcal{E}_{\epsilon}[g] .
$$

By Lemma 6.1, (A.5), (A.6) and (6.6),

$$
\begin{aligned}
\left|\int_{\Omega}(a+\epsilon V)\left(2 r g+g^{2}\right) d y\right| & \leq\|a+\epsilon V\|_{\infty}\|g\|_{6 / 5}\left(2\|r\|_{6}+\|g\|_{6}\right) \\
& \lesssim\left(|\beta| \lambda^{-1}\left\|P U_{x, \lambda}\right\|_{6 / 5}+|\gamma|\left\|\partial_{\lambda} P U_{x, \lambda}\right\|_{6 / 5}\right)\left(\|r\|_{6}+\|g\|_{6}\right) \\
& =o\left(\lambda^{-2}\right) .
\end{aligned}
$$

We have, since $r \in T_{x, \lambda}^{\perp}$ and $g \in T_{x, \lambda}$,

$$
\int_{\Omega} \nabla r \cdot \nabla g d y=0
$$

This proves (6.9).

Step 3 We finally extract the relevant contribution from $r$ and show

$$
\mathcal{E}_{\epsilon}\left[\psi_{x, \lambda}+r\right]=\mathcal{E}_{\epsilon}\left[\psi_{x, \lambda}\right]+\mathcal{E}_{0}[r]+o\left(\lambda^{-2}\right)+o\left(\epsilon \lambda^{-1}\right) .
$$

Indeed,

$$
\mathcal{E}_{\epsilon}\left[\psi_{x, \lambda}+r\right]=\mathcal{E}_{\epsilon}\left[\psi_{x, \lambda}\right]+2 \mathcal{E}_{\epsilon}\left[\psi_{x, \lambda}, r\right]+\mathcal{E}_{\epsilon}[r] .
$$

Using $r \in T_{x, \lambda}^{\perp}$, the harmonicity of $H_{0}$ and equation (2.11) for $H_{a}$, we find

$$
\begin{aligned}
& \int_{\Omega} \nabla \psi_{x, \lambda} \cdot \nabla r d y=-\lambda^{-1 / 2} \int_{\Omega} \nabla_{y}\left(H_{a}(x, y)-H_{0}(x, y)\right) \cdot \nabla r d y \\
& \quad=-\lambda^{-1 / 2} \int_{\Omega} a G_{a}(x, y) r d y .
\end{aligned}
$$


On the other hand, by (2.17), (2.18) and (4.2),

$$
\int_{\Omega} a \psi_{x, \lambda} r d y-\int_{\Omega} a U_{x, \lambda} r d y+\lambda^{-1 / 2} \int_{\Omega} a H_{a}(x, y) r d y=\mathcal{O}\left(\|a\|_{6 / 5}\left\|f_{x, \lambda}\right\|_{\infty}\|r\|_{6}\right)=o\left(\lambda^{-3}\right) .
$$

Thus,

$$
\mathcal{E}_{0}\left[\psi_{x, \lambda}, r\right]=\int_{\Omega} a\left(U_{x, \lambda}-\lambda^{-1 / 2} H_{a}(x, y)-\lambda^{-1 / 2} G_{a}(x, y)\right) r d y+o\left(\lambda^{-3}\right) .
$$

By Lemma 2.4,

$$
\begin{aligned}
& \left|\int_{\Omega} a\left(U_{x, \lambda}-\lambda^{-1 / 2} H_{a}(x, y)-\lambda^{-1 / 2} G_{a}(x, y)\right) r d y\right| \\
& \quad \leq\|a\|_{\infty}\left\|U_{x, \lambda}-\lambda^{-1 / 2} H_{a}(x, \cdot)-\lambda^{-1 / 2} G_{a}(x, \cdot)\right\|_{6 / 5}\|r\|_{6}=o\left(\lambda^{-5 / 2}\right) .
\end{aligned}
$$

Finally, by (6.6) and (A.8),

$$
\left|\int_{\Omega} V \psi_{x, \lambda} r d y\right| \leq\|V\|_{\infty}\left\|\psi_{x, \lambda}\right\|_{6 / 5}\|r\|_{6}=o\left(\lambda^{-1}\right)
$$

and

$$
\left|\int_{\Omega} V r^{2} d y\right| \leq\|V\|_{3 / 2}\|r\|_{6}^{2}=o\left(\lambda^{-1}\right)
$$

This proves (6.10).

The lemma follows by collecting the estimates from the three steps.

Expanding the denominator Recall that $D_{0}$ and $\mathcal{I}[r]$ were defined in (6.4) and (6.5) respectively. We shall show

Lemma 6.5 As $\epsilon \rightarrow 0$,

$$
\alpha^{-6} \int_{\Omega} u_{\epsilon}^{6} d y=D_{0}+D_{1}+\mathcal{I}[r]+o\left(\lambda^{-2}\right),
$$

where

$$
D_{1}:=6 \int_{\Omega} \psi_{x, \lambda}^{5} g d y+15 \int_{\Omega} \psi_{x, \lambda}^{4} g^{2} d y
$$

Proof Step 1 We show that the contribution from $h$ to $\alpha^{-6} \int_{\Omega} u_{\epsilon}^{6} d y$ is negligible, that is,

$$
\alpha^{-6} \int_{\Omega} u_{\epsilon}^{6} d y=\int_{\Omega}\left(\psi_{x, \lambda}+g+r\right)^{6} d y+o\left(\lambda^{-2}\right) .
$$

Indeed,

$$
\begin{aligned}
\alpha^{-6} \int_{\Omega} u_{\epsilon}^{6} d y= & \int_{\Omega}\left(\psi_{x, \lambda}+g+r\right)^{6} d y+6 \int_{\Omega}\left(\psi_{x, \lambda}+g+r\right)^{5} h d y \\
& +\mathcal{O}\left(\left\|\psi_{x, \lambda}+g+r\right\|_{6}^{4}\|h\|_{6}^{2}+\|h\|_{6}^{6}\right)
\end{aligned}
$$

and by (6.6) the last term is $\mathcal{O}\left(\lambda^{-4}\right)$. The middle term is

$$
\int_{\Omega}\left(\psi_{x, \lambda}+g+r\right)^{5} h d y=\int_{\Omega} \psi_{x, \lambda}^{5} h d y+\mathcal{O}\left(\left\|\psi_{x, \lambda}\right\|_{6}^{4}\|g+r\|_{6}\|h\|_{6}+\|g+r\|_{6}^{5}\|h\|_{6}\right)
$$


and again by (6.6) the last term here is $o\left(\lambda^{-5 / 2}\right)$. The first term here is

$$
\int_{\Omega} \psi_{x, \lambda}^{5} h d y=\int_{\Omega} U_{x, \lambda}^{5} h d y+\mathcal{O}\left(\left\|U_{x, \lambda}\right\|_{6}^{4}\left\|\psi_{x, \lambda}-U_{\lambda, x}\right\|_{6}\|h\|_{6}+\left\|\psi_{x, \lambda}-U_{x, \lambda}\right\|_{6}^{5}\|h\|_{6}\right),
$$

which, by (6.6) and (A.7), is $\mathcal{O}\left(\lambda^{-5 / 2}\right)$. Finally, by (5.12) and (5.10),

$$
\int_{\Omega} U_{x, \lambda}^{5} h d y=3^{-1} \int_{\Omega} \nabla P U_{x, \lambda} \cdot \nabla h d y=\sum_{j=1}^{3} \delta_{j} \lambda^{-3} \int_{\Omega} \nabla P U_{x, \lambda} \cdot \nabla \partial_{x_{j}} P U_{x, \lambda} d y=\mathcal{O}\left(\lambda^{-3}\right) .
$$

This proves (6.11).

Step 2 We now extract the relevant contribution from $g$ and show

$$
\int_{\Omega}\left(\psi_{x, \lambda}+g+r\right)^{6} d y=\int_{\Omega}\left(\psi_{x, \lambda}+r\right)^{6} d y+6 \int_{\Omega} \psi_{x, \lambda}^{5} g d y+15 \int_{\Omega} \psi_{x, \lambda}^{4} g^{2} d y+o\left(\lambda^{-2}\right) .
$$

Indeed,

$$
\begin{aligned}
\int_{\Omega}\left(\psi_{x, \lambda}+g+r\right)^{6} d y= & \int_{\Omega}\left(\psi_{x, \lambda}+r\right)^{6} d y+6 \int_{\Omega}\left(\psi_{x, \lambda}+r\right)^{5} g d y+15 \int_{\Omega}\left(\psi_{x, \lambda}+r\right)^{4} g^{2} d y \\
& +\mathcal{O}\left(\left\|\psi_{x, \lambda}+r\right\|_{6}^{3}\|g\|_{6}^{3}+\|g\|_{6}^{6}\right)
\end{aligned}
$$

and by (6.6) the last term is $\mathcal{O}\left(\lambda^{-3}\right)$. We need to show that the contribution from $r$ to the second and third term on the right side is negligible. The third term is

$$
\int_{\Omega}\left(\psi_{x, \lambda}+r\right)^{4} g^{2} d y=\int_{\Omega} \psi_{x, \lambda}^{4} g^{2} d y+\mathcal{O}\left(\left\|\psi_{x, \lambda}\right\|_{6}^{3}\|r\|_{6}\|g\|_{6}^{2}+\|r\|_{6}^{4}\|g\|_{6}^{2}\right)
$$

and by (6.6) the last term is $o\left(\lambda^{-5 / 2}\right)$. The second term above is

$$
\int_{\Omega}\left(\psi_{x, \lambda}+r\right)^{5} g d y=\int_{\Omega} \psi_{x, \lambda}^{5} g d y+5 \int_{\Omega} \psi_{x, \lambda}^{4} r g d y+\mathcal{O}\left(\left\|\psi_{x, \lambda}\right\|_{6}^{3}\|r\|_{6}^{2}\|g\|_{6}+\|r\|_{6}^{5}\|g\|_{6}^{2}\right)
$$

and by (6.6) the last term is $o\left(\lambda^{-2}\right)$. Let us show that the second term on the right side of the previous equation is negligible. We have

$$
\begin{aligned}
& \int_{\Omega} \psi_{x, \lambda}^{4} r g d y \\
& \quad=\int_{\Omega} U_{x, \lambda}^{4} r g d y+\mathcal{O}\left(\left\|U_{x, \lambda}\right\|_{6}^{3}\left\|\psi_{x, \lambda}-U_{x, \lambda}\right\|_{6}\|r\|_{6}\|g\|_{6}+\left\|\psi_{x, \lambda}-U_{x, \lambda}\right\|_{6}^{4}\|r\|_{6}\left\|_{6}\right\|_{6}\right)
\end{aligned}
$$

and by (6.6) and (A.7) the last term is $o\left(\lambda^{-2}\right)$. Now

$$
\begin{aligned}
\int_{\Omega} U_{x, \lambda}^{4} r g d y= & \beta \lambda^{-1} \int_{\Omega} U_{x, \lambda}^{4} P U_{x, \lambda} r d y+\gamma \int_{\Omega} U_{x, \lambda}^{4} \partial_{\lambda} P U_{x, \lambda} r d y \\
= & \beta \lambda^{-1} \int_{\Omega} U_{x, \lambda}^{5} r d y+\gamma \int_{\Omega} U_{x, \lambda}^{4} \partial_{\lambda} U_{x, \lambda} r d y \\
& +\mathcal{O}\left(\left(|\beta| \lambda^{-1}\left\|P U_{x, \lambda}-U_{x, \lambda}\right\|_{6}+|\gamma|\left\|\partial_{\lambda} P U_{x, \lambda}-\partial_{\lambda} U_{x, \lambda}\right\|_{6}\right)\left\|U_{x, \lambda}\right\|_{6}^{4}\|r\|_{6}\right) .
\end{aligned}
$$


By Lemma 6.1, [24, Prop. 1 (c)] and (6.6), the last term is $o\left(\lambda^{-2}\right)$. Finally, by (5.19) and the fact that $r \in T_{x, \lambda}^{\perp}$,

$$
\begin{aligned}
& \int_{\Omega} U_{x, \lambda}^{5} r d y=3^{-1} \int_{\Omega} \nabla P U_{x, \lambda} \cdot \nabla r d y=0, \quad \int_{\Omega} U_{x, \lambda}^{4} \partial_{\lambda} U_{x, \lambda} r d y \\
& =(15)^{-1} \int_{\Omega} \nabla \partial_{\lambda} P U_{x, \lambda} \cdot \nabla r d y=0 .
\end{aligned}
$$

This proves (6.12).

Step 3 We finally extract the relevant contribution from $r$ and show

$$
\int_{\Omega}\left(\psi_{x, \lambda}+r\right)^{6} d y=\int_{\Omega} \psi_{x, \lambda}^{6} d y+\mathcal{I}[r]+o\left(\lambda^{-2}\right) .
$$

Indeed,

$$
\begin{aligned}
\int_{\Omega}\left(\psi_{x, \lambda}+r\right)^{6} d y= & \int_{\Omega} \psi_{x, \lambda}^{6} d y+6 \int_{\Omega} \psi_{x, \lambda}^{5} r d y+15 \int_{\Omega} \psi_{x, \lambda}^{4} r^{2} d y+20 \int_{\Omega} \psi_{x, \lambda}^{3} r^{3} d y \\
& +\mathcal{O}\left(\left\|\psi_{x, \lambda}\right\|_{6}^{2}\|r\|_{6}^{4}+\|r\|_{6}^{6}\right)
\end{aligned}
$$

and by (6.6) the last term is $o\left(\lambda^{-2}\right)$. We need to extract $\mathcal{I}[r]$ from the three terms on the right side involving $r$. We begin with the term that is linear in $r$,

$$
\begin{aligned}
\int_{\Omega} \psi_{x, \lambda}^{5} r d y= & \int_{\Omega} U_{x, \lambda}^{5} r d y+5 \int_{\Omega} U_{x, \lambda}^{4}\left(\psi_{x, \lambda}-U_{x, \lambda}\right) r d y \\
& +\mathcal{O}\left(\left\|U_{x, \lambda}\right\|_{18 / 5}^{3}\left\|\psi_{x, \lambda}-U_{x, \lambda}\right\|_{\infty}\|r\|_{6}+\left\|\psi_{x, \lambda}-U_{x, \lambda}\right\|_{6}^{5}\|r\|_{6}\right) .
\end{aligned}
$$

By (A.7), (6.6) and $\left\|U_{x, \lambda}\right\|_{18 / 5}^{3}=\mathcal{O}\left(\lambda^{-1}\right)$, the last term is $o\left(\lambda^{-2}\right)$. Since $r \in T_{x, \lambda}^{\perp}$, the first term is

$$
\int_{\Omega} U_{x, \lambda}^{5} r d y=3^{-1} \int_{\Omega} \nabla P U_{x, \lambda} \cdot \nabla r d y=0
$$

Writing $\psi_{x, \lambda}-U_{x, \lambda}=-\lambda^{-1 / 2} H_{a}(x, \cdot)-f_{x, \lambda}$, we have

$$
\int_{\Omega} U_{x, \lambda}^{4}\left(\psi_{x, \lambda}-U_{x, \lambda}\right) r d y=-\lambda^{-1 / 2} \int_{\Omega} U_{x, \lambda}^{4} H_{a}(x, y) r d y+\mathcal{O}\left(\left\|U_{x, \lambda}\right\|_{24 / 5}^{4}\left\|f_{x, \lambda}\right\|_{\infty}\|r\|_{6}\right) .
$$

By (2.18), (4.2), (6.6) and $\left\|U_{x, \lambda}\right\|_{24 / 5}^{4}=\mathcal{O}\left(\lambda^{-1 / 2}\right)$, the last term on the right side is $o\left(\lambda^{-2}\right)$.

We now turn to the terms that are quadratic in $r$. We have

$\int_{\Omega} \psi_{x, \lambda}^{4} r^{2} d y=\int_{\Omega} U_{x, \lambda}^{4} r^{2} d y+\mathcal{O}\left(\left\|U_{x, \lambda}\right\|_{9 / 2}^{3}\left\|\psi_{x, \lambda}-U_{x, \lambda}\right\|_{\infty}\|r\|_{6}^{2}+\left\|\psi_{x, \lambda}-U_{x, \lambda}\right\|_{6}^{4}\|r\|_{6}^{2}\right)$

and by (A.7), (6.6) and $\left\|U_{x, \lambda}\right\|_{9 / 2}^{3}=\mathcal{O}\left(\lambda^{-1 / 2}\right)$, the last term on the right side is $o\left(\lambda^{-2}\right)$. Similarly, one shows that

$$
\int_{\Omega} \psi_{x, \lambda}^{3} r^{3} d y=\int_{\Omega} U_{x, \lambda}^{3} r^{3} d y+o\left(\lambda^{-2}\right) .
$$

This proves (6.13).

The lemma follows by collecting the estimates from the three steps. 
Proof of Lemma 6.3 Note that, by (6.6), $D_{1}=\mathcal{O}\left(\lambda^{-1}\right)$ and $\mathcal{I}[r]=o\left(\lambda^{-1}\right)$. Moreover, by (2.3), $D_{0}$ stays away from zero. Therefore, the expansion from Lemma 6.5 implies that

$$
\left(\alpha^{-6} \int_{\Omega} u_{\epsilon}^{6} d y\right)^{-1 / 3}=D_{0}^{-1 / 3}\left(1-\frac{1}{3} \frac{D_{1}}{D_{0}}-\frac{1}{3} \frac{\mathcal{I}[r]}{D_{0}}+\frac{2}{9} \frac{D_{1}^{2}}{D_{0}^{2}}+o\left(\lambda^{-2}\right)\right) .
$$

Combining this with the expansion from Lemma 6.4 and using $N_{1}=\mathcal{O}\left(\lambda^{-1}\right)$ (again from (6.6)), we obtain

$$
\mathcal{S}_{a+\epsilon V}\left[u_{\epsilon}\right]=\mathcal{S}_{a+\epsilon V}\left[\psi_{x, \lambda}\right]+A+D_{0}^{-1 / 3}\left(\mathcal{E}_{0}[r]-\frac{N_{0}}{3 D_{0}} \mathcal{I}[r]\right)+o\left(\lambda^{-2}\right)+o\left(\epsilon \lambda^{-1}\right)
$$

with

$$
A=D_{0}^{-1 / 3}\left(N_{1}-\frac{D_{1}}{3 D_{0}} N_{1}-\frac{D_{1}}{3 D_{0}} N_{0}+\frac{2}{9} \frac{D_{1}^{2}}{D_{0}^{2}} N_{0}\right)
$$

Thus, the assertion of the lemma is equivalent to $A=o\left(\lambda^{-2}\right)+o\left(\epsilon \lambda^{-1}\right)$. We write

$$
A=D_{0}^{-1 / 3}\left(\left(N_{1}-D_{1}\right)\left(1-\frac{D_{1}}{3 D_{0}}\right)+\frac{1}{3} \frac{D_{1}^{2}}{D_{0}}+\left(1-\frac{N_{0}}{3 D_{0}}\right) D_{1}\left(1-\frac{2 D_{1}}{3 D_{0}}\right)\right) .
$$

It follows from (2.2) and (2.3) that

$$
\frac{N_{0}}{3 D_{0}}=1+\mathcal{O}\left(\lambda^{-2}\right)+\mathcal{O}\left(\epsilon \lambda^{-1}\right) .
$$

This, together with $D_{1}=\mathcal{O}\left(\lambda^{-1}\right)$, yields

$$
A=D_{0}^{-1 / 3}\left(\left(N_{1}-D_{1}\right)\left(1-\frac{D_{1}}{3 D_{0}}\right)+\frac{1}{3} \frac{D_{1}^{2}}{D_{0}}\right)+o\left(\lambda^{-2}\right) .
$$

We shall show in Appendix A that

$$
N_{1}=\frac{3 \pi^{2}}{2} \beta \lambda^{-1}+\left(\frac{3 \pi^{2}}{4} \beta^{2}+\frac{15 \pi^{2}}{64} \gamma^{2}-8 \pi \phi_{0}(x) \beta+4 \pi \phi_{0}(x) \gamma\right) \lambda^{-2}+o\left(\lambda^{-2}\right)
$$

and

$$
D_{1}=\frac{3 \pi^{2}}{2} \beta \lambda^{-1}+\left(\frac{15 \pi^{2}}{4} \beta^{2}+\frac{15 \pi^{2}}{64} \gamma^{2}-8 \pi \phi_{0}(x) \beta+4 \pi \phi_{0}(x) \gamma\right) \lambda^{-2}+o\left(\lambda^{-2}\right) .
$$

Thus, in particular,

$$
N_{1}-D_{1}=-3 \pi^{2} \beta^{2} \lambda^{-2}+o\left(\lambda^{-2}\right) \quad \text { and } \quad D_{1}^{2}=\left(\frac{3 \pi^{2}}{2}\right)^{2} \beta^{2} \lambda^{-2}+o\left(\lambda^{-2}\right) .
$$

This, together with $D_{0}=(S / 3)^{3 / 2}+o\left(\lambda^{-1}\right)$ (from (2.3)), implies $A=o\left(\lambda^{-2}\right)$, as claimed. 
Before continuing with the main line of the argument, let us expand $\alpha$. By the normalization (1.10), Lemma 6.5, (2.3) and (6.16)

$$
\begin{aligned}
\alpha^{-6}(S / 3)^{3 / 2}= & (S / 3)^{3 / 2}+\frac{3 \pi^{2}}{2} \beta \lambda^{-1}-8 \pi \phi_{a}(x) \lambda^{-1} \\
& +\left(8 \pi a(x)+\frac{15 \pi^{2}}{4} \beta^{2}+\frac{15 \pi^{2}}{64} \gamma^{2}-8 \pi \phi_{0}(x) \beta+4 \pi \phi_{0}(x) \gamma\right) \lambda^{-2} \\
& +\mathcal{I}[r]+o\left(\lambda^{-2}\right) .
\end{aligned}
$$

\subsection{Coercivity}

To complete the proof of our main results, it remains to prove that the terms involving $r$ in the expansion (6.3) give a non-negative contribution. Recall that $\mathcal{I}[r]$ was defined in (6.5) and $N_{0}$ and $D_{0}$ in Lemmas 6.4 and 6.5, respectively.

Lemma 6.6 There is a $\rho>0$ such that for all sufficiently small $\epsilon>0$,

$$
\mathcal{E}_{0}[r]-\frac{N_{0}}{3 D_{0}} \mathcal{I}[r] \geq \rho \int_{\Omega}|\nabla r|^{2} d y+o\left(\lambda^{-2}\right) .
$$

Proof We bound, using (4.2), Lemma 2.6 and (5.1), for any $\delta>0$,

$$
\begin{aligned}
& \left|30 \lambda^{-1 / 2} \int_{\Omega} U_{x, \lambda}^{4} H_{a}(x, y) r d y\right| \leq 30 \lambda^{-1 / 2}\left(\int_{\Omega} U_{x, \lambda}^{4} r^{2} d y\right)^{\frac{1}{2}}\left(\int_{\Omega} U_{x, \lambda}^{4} H_{a}(x, y)^{2} d y\right)^{\frac{1}{2}} \\
& \leq o\left(\lambda^{-1}\right)\left(\int_{\Omega} U_{x, \lambda}^{4} r^{2} d y\right)^{\frac{1}{2}} \leq \delta \int_{\Omega} U_{x, \lambda}^{4} r^{2} d y+\delta^{-1} o\left(\lambda^{-2}\right) .
\end{aligned}
$$

Similarly, using (6.6),

$$
\begin{aligned}
& \left|20 \int_{\Omega} U_{x, \lambda}^{3} r^{3} d y\right| \leq 20\left(\int_{\Omega} U_{x, \lambda}^{4} r^{2} d y\right)^{\frac{3}{4}}\left(\int_{\Omega} r^{6} d y\right)^{\frac{1}{4}} \leq o\left(\lambda^{-\frac{3}{4}}\right)\left(\int_{\Omega} U_{x, \lambda}^{4} r^{2} d y\right)^{\frac{3}{4}} \\
& \quad \leq \delta \int_{\Omega} U_{x, \lambda}^{4} r^{2} d y+\delta^{-3} o\left(\lambda^{-3}\right) .
\end{aligned}
$$

This, together with (6.14) implies that

$$
\begin{aligned}
\mathcal{E}_{0}[r]-\frac{N_{0}}{3 D_{0}} \mathcal{I}[r] \geq \int_{\Omega}\left(|\nabla r|^{2}+a r^{2}-15 U_{x, \lambda}^{4} r^{2}\right) d y \\
-\left(2 \delta+\mathcal{O}\left(\lambda^{-2}\right)+\mathcal{O}\left(\epsilon \lambda^{-1}\right)\right) \int_{\Omega} U_{x, \lambda}^{4} r^{2} d y+\delta^{-1} o\left(\lambda^{-2}\right)+\delta^{-3} o\left(\lambda^{-3}\right) .
\end{aligned}
$$

Since $r \in T_{x, \lambda}^{\perp}$, Lemma 4.3 implies that for all sufficiently small $\epsilon>0$, the first term on the right side is bounded from below by $\rho \int_{\Omega}|\nabla r|^{2} d y$ for some $\rho>0$ independent of $\epsilon$. On the other hand, by (5.20), choosing $\delta>0$ small, but independent of $\epsilon$, and then $\epsilon$ small, we can make sure that

$$
-\left(2 \delta+\mathcal{O}\left(\lambda^{-2}\right)+\mathcal{O}\left(\epsilon \lambda^{-1}\right)\right) \int_{\Omega} U_{x, \lambda}^{4} r^{2} d y \geq-(\rho / 2) \int_{\Omega}|\nabla r|^{2} d y .
$$

This completes the proof of the lemma. 


\subsection{Proof of the main results}

In this subsection we prove Theorems 1.3, 1.4 and 1.7. Combining the expansions from Lemma 6.3 and Theorem 2.1 and using the fact that $\phi_{a}\left(x_{0}\right)=0$ (see Proposition 5.1) we obtain

$$
\begin{aligned}
& \mathcal{S}_{a+\epsilon V}\left[u_{\epsilon}\right] \geq S+(S / 3)^{-1 / 2}\left(\frac{\epsilon}{\lambda} Q_{V}\left(x_{0}\right)-\frac{2 \pi^{2} a\left(x_{0}\right)}{\lambda^{2}}\right) \\
& +(S / 3)^{-1 / 2} 4 \pi \phi_{a}(x) \lambda^{-1}+(S / 3)^{-1 / 2}\left(\mathcal{E}_{0}[r]-\frac{N_{0}}{3 D_{0}} \mathcal{I}[r]\right)+o\left(\lambda^{2}\right)+o\left(\epsilon \lambda^{-1}\right) .
\end{aligned}
$$

Using the almost minimizing assumption (1.10) as well as the coercivity bound from Lemma 6.6 we obtain

$$
\begin{aligned}
0 \geq & (1+o(1))(S-S(a+\epsilon V))+(S / 3)^{-1 / 2}\left(\frac{\epsilon}{\lambda} Q_{V}\left(x_{0}\right)-\frac{2 \pi^{2} a\left(x_{0}\right)}{\lambda^{2}}\right)+\mathcal{R}+o\left(\lambda^{2}\right) \\
& +o\left(\epsilon \lambda^{-1}\right) .
\end{aligned}
$$

with

$$
\mathcal{R}:=(S / 3)^{-1 / 2}\left(4 \pi \phi_{a}(x) \lambda^{-1}+\rho \int_{\Omega}|\nabla r|^{2} d y\right) .
$$

Note that, by Corollary 2.2, $\mathcal{R} \geq 0$.

Lemma 6.7 If $\mathcal{N}_{a}(V) \neq \emptyset$, then $x_{0} \in \mathcal{N}_{a}(V)$.

This is the only place in the proof of Theorem 1.3 where we need assumption (1.4).

Proof We recall the upper bound from Corollary 2.3,

$$
S(a+\epsilon V) \leq S-(S / 3)^{-1 / 2} \sup _{y \in \mathcal{N}_{a}(V)} \frac{Q_{V}(y)^{2}}{8 \pi^{2}|a(y)|} \epsilon^{2}+o\left(\epsilon^{2}\right) .
$$

Combining this with (6.18) and using $\mathcal{R} \geq 0$, we find

$$
C_{1} \epsilon^{2}+C_{2} \lambda^{-2} \leq\left(-(S / 3)^{-1 / 2} Q_{V}\left(x_{0}\right)+o(1)\right) \frac{\epsilon}{\lambda}
$$

with

$$
C_{1}:=(S / 3)^{-1 / 2} \sup _{y \in \mathcal{N}_{a}(V)} \frac{Q_{V}(y)^{2}}{8 \pi^{2}|a(y)|}+o(1), \quad C_{2}:=(S / 3)^{-1 / 2} 2 \pi^{2}\left|a\left(x_{0}\right)\right|+o(1) .
$$

By the assumptions $\mathcal{N}_{a}(V) \neq \varnothing$ and (1.4), both $C_{1}$ and $C_{2}$ tend to some positive quantities as $\epsilon \rightarrow 0$. Since $C_{1} \epsilon^{2}+C_{2} \lambda^{-2} \geq 2 \sqrt{C_{1} C_{2}} \epsilon \lambda^{-1}$ we obtain that $Q_{V}\left(x_{0}\right)<0$, as claimed. 
We now assume $\mathcal{N}_{a}(V) \neq \varnothing$ and complete the proof of Theorems 1.3 and 1.7. We can write

$$
\begin{aligned}
& (S / 3)^{-1 / 2}\left(\frac{\epsilon}{\lambda} Q_{V}\left(x_{0}\right)-\frac{2 \pi^{2} a\left(x_{0}\right)}{\lambda^{2}}\right)+o\left(\lambda^{2}\right)+o\left(\epsilon \lambda^{-1}\right) \\
& =-(S / 3)^{-1 / 2} \frac{\left(Q_{V}\left(x_{0}\right)+o(1)\right)^{2}}{4\left(2 \pi^{2}\left|a\left(x_{0}\right)\right|+o(1)\right)} \epsilon^{2} \\
& \quad+(S / 3)^{-1 / 2}\left(\frac{Q_{V}\left(x_{0}\right)+o(1)}{2 \sqrt{2 \pi^{2}\left|a\left(x_{0}\right)\right|+o(1)}} \epsilon+\sqrt{2 \pi^{2}\left|a\left(x_{0}\right)\right|+o(1)} \lambda^{-1}\right)^{2} .
\end{aligned}
$$

Inserting this into (6.18) we obtain

$$
(S / 3)^{-1 / 2} \frac{\left(Q_{V}\left(x_{0}\right)+o(1)\right)^{2}}{4\left(2 \pi^{2}\left|a\left(x_{0}\right)\right|+o(1)\right)} \epsilon^{2} \geq(1+o(1))(S-S(a+\epsilon V))+\mathcal{R}^{\prime}
$$

with

$$
\mathcal{R}^{\prime}:=\mathcal{R}+(S / 3)^{-1 / 2}\left(\frac{Q_{V}\left(x_{0}\right)+o(1)}{2 \sqrt{2 \pi^{2}\left|a\left(x_{0}\right)\right|+o(1)}} \epsilon+\sqrt{2 \pi^{2}\left|a\left(x_{0}\right)\right|+o(1)} \lambda^{-1}\right)^{2} .
$$

Since $\mathcal{R}^{\prime} \geq 0$ we obtain, in particular,

$$
\begin{aligned}
S-S(a+\epsilon V) & \leq(1+o(1))(S / 3)^{-1 / 2} \frac{\left(Q_{V}\left(x_{0}\right)+o(1)\right)^{2}}{4\left(2 \pi^{2}\left|a\left(x_{0}\right)\right|+o(1)\right)} \epsilon^{2} \\
& =(S / 3)^{-1 / 2} \frac{Q_{V}\left(x_{0}\right)^{2}}{8 \pi^{2}\left|a\left(x_{0}\right)\right|} \epsilon^{2}+o\left(\epsilon^{2}\right) \\
& \leq(S / 3)^{-1 / 2} \sup _{y \in \mathcal{N}_{a}(V)} \frac{Q_{V}(y)^{2}}{8 \pi^{2}|a(y)|} \epsilon^{2}+o\left(\epsilon^{2}\right) .
\end{aligned}
$$

In the last inequality we used $x_{0} \in \mathcal{N}_{a}(V)$. This proves the claimed lower bound on $S(a+\epsilon V)$ and completes the proof of Theorem 1.3.

We now proceed to the proof of Theorem 1.7, still under the assumption $\mathcal{N}_{a}(V) \neq \varnothing$. Combining the lower bound on $S-S(a+\epsilon V)$ from Corollary 2.3 with the upper bound in (6.22) we obtain

$$
\frac{Q_{V}\left(x_{0}\right)^{2}}{\left|a\left(x_{0}\right)\right|}=\sup _{y \in \mathcal{N}_{a}(V)} \frac{Q_{V}(y)^{2}}{|a(y)|} .
$$

Moreover, inserting the lower bound on $S-S(a+\epsilon V)$ into (6.20) we infer that $\mathcal{R}^{\prime}=o\left(\epsilon^{2}\right)$. Thus, by (6.19) and (6.21)

$$
\|\nabla r\|^{2}=o\left(\epsilon^{2}\right) \quad \text { and } \quad \lambda^{-1}=\frac{\left|Q_{V}\left(x_{0}\right)\right|}{4 \pi^{2}\left|a\left(x_{0}\right)\right|} \epsilon+o(\epsilon) .
$$

and, reinserting the last expression into $\mathcal{R}=o\left(\epsilon^{2}\right)$, also

$$
\phi_{a}(x)=o(\epsilon) .
$$


Inserting these bounds into (6.17), we obtain

$$
\begin{aligned}
\alpha^{-6}= & 1+(S / 3)^{-3 / 2} \frac{3 \pi}{2} \beta \lambda^{-1} \\
& +(S / 3)^{-3 / 2}\left(8 \pi a\left(x_{0}\right)+\frac{15 \pi^{2}}{4} \beta^{2}+\frac{15 \pi^{2}}{64} \gamma^{2}-8 \pi \phi_{0}\left(x_{0}\right) \beta+4 \pi \phi_{0}\left(x_{0}\right) \gamma\right) \lambda^{-2} \\
& +o\left(\epsilon^{2}\right)
\end{aligned}
$$

and therefore, using Lemma 6.1, $\alpha=1+\mathcal{O}(\epsilon)$. This completes the proof of Theorem 1.7.

We now assume $\mathcal{N}_{a}(V)=\emptyset$ and prove Theorem 1.4. Estimating $Q_{V}\left(x_{0}\right) \geq 0$ and $\mathcal{R} \geq 0$ in (6.18) we obtain

$$
0 \geq(1+o(1))(S-S(a+\epsilon V))+\left((S / 3)^{-1 / 2} 2 \pi^{2}\left|a\left(x_{0}\right)\right|+o(1)\right) \lambda^{-2}+o\left(\epsilon \lambda^{-1}\right) .
$$

Since $o\left(\epsilon \lambda^{-1}\right) \geq-\delta \lambda^{-2}+o\left(\epsilon^{2}\right)$ for any fixed $\delta$, this implies $S-S(a+\epsilon V)=o\left(\epsilon^{2}\right)$.

Under the additional assumption $Q_{V}\left(x_{0}\right)>0$, we infer from (6.18) that

$$
0 \geq(1+o(1))(S-S(a+\epsilon V))+C_{1} \epsilon \lambda^{-1}+C_{2} \lambda^{-2}
$$

with

$$
C_{1}:=(S / 3)^{-1 / 2} Q_{V}\left(x_{0}\right)+o(1) \text { and } C_{2}:=(S / 3)^{-1 / 2} 2 \pi^{2}\left|a\left(x_{0}\right)\right|+o(1) .
$$

Since both $C_{1}$ and $C_{2}$ are positive for all sufficiently small $\epsilon>0$, we arrive at a contradiction. Thus, assumption (3.4), under which we have worked so far, is not satisfied. By the concavity argument in the proof of Corollary 2.3 this means that $S(a+\epsilon V)=S$ for all sufficiently small $\epsilon>0$. This concludes the proof of Theorem 1.4.

Funding Open Access funding enabled and organized by Projekt DEAL.

Open Access This article is licensed under a Creative Commons Attribution 4.0 International License, which permits use, sharing, adaptation, distribution and reproduction in any medium or format, as long as you give appropriate credit to the original author(s) and the source, provide a link to the Creative Commons licence, and indicate if changes were made. The images or other third party material in this article are included in the article's Creative Commons licence, unless indicated otherwise in a credit line to the material. If material is not included in the article's Creative Commons licence and your intended use is not permitted by statutory regulation or exceeds the permitted use, you will need to obtain permission directly from the copyright holder. To view a copy of this licence, visit http://creativecommons.org/licenses/by/4.0/.

\section{Appendix A : Some computations}

\section{A.1 Asymptotics and bounds}

We recall that we abbreviate $d=\operatorname{dist}(x, \partial \Omega)$.

Lemma A.1 As $\lambda \rightarrow \infty$, uniformly in $x \in \Omega$,

$$
\begin{aligned}
& \int_{\Omega}\left|\nabla P U_{x, \lambda}\right|^{2} d y=3^{-1 / 2} S^{3 / 2}-4 \pi \phi_{0}(x) \lambda^{-1}+o\left((\lambda d)^{-1}\right), \\
& \int_{\Omega} P U_{x, \lambda}^{6} d y=(S / 3)^{3 / 2}-8 \pi \phi_{0}(x) \lambda^{-1}+o\left((\lambda d)^{-1}\right) .
\end{aligned}
$$


Proof We set again $\phi_{x, \lambda}=U_{x, \lambda}-P U_{x, \lambda}$. Then, by (1.9) and (2.15),

$$
\begin{aligned}
& \int_{\Omega}\left|\nabla P_{x, \lambda}\right|^{2} d y=\int_{\Omega} \nabla P U_{x, \lambda} \cdot \nabla U_{x, \lambda} d y=3 \int_{\Omega} P U_{x, \lambda} U_{x, \lambda}^{5} d y \\
& \quad=3 \int_{\Omega} U_{x, \lambda}^{6} d y-3 \int_{\Omega} U_{x, \lambda}^{5} \phi_{x, \lambda} d y .
\end{aligned}
$$

By [24, Proof of (B.3)]

$$
\int_{\Omega} U_{x, \lambda}^{6} d y=(S / 3)^{3 / 2}+o\left((d \lambda)^{-1}\right)
$$

and, as shown in [15, Proof of Thm.1.1],

$$
\int_{\Omega} U_{x, \lambda}^{5} \phi_{x, \lambda} d y=\frac{4 \pi}{3} \phi_{0}(x) \lambda^{-1}+o\left((d \lambda)^{-1}\right) .
$$

(Since $\phi_{x, \lambda}=\lambda^{-1 / 2} H_{0}(x, \cdot)+f_{x, \lambda}$, the proof of the latter relation is similar to the proof of Lemma 2.5, but to get the uniformity even for $x$ close to the boundary more careful bounds on $\nabla_{y} H_{0}(x, y)$ are needed.) This proves (A.1).

To prove (A.2), we write

$$
\int_{\Omega} P U_{x, \lambda}^{6} d y=\int_{\Omega} U_{x, \lambda}^{6} d y-6 \int_{\Omega} U_{x, \lambda}^{5} \phi_{x, \lambda} d y+\mathcal{O}\left(\left\|U_{x, \lambda}\right\|_{4}^{4}\left\|\phi_{x, \lambda}\right\|_{\infty}^{2}+\left\|\phi_{x, \lambda}\right\|_{6}^{6}\right) .
$$

For the first two terms we use (A.3), (A.4). Moreover, $\left\|\phi_{x, \lambda}\right\|_{\infty}=\mathcal{O}\left(\lambda^{-1 / 2} d^{-1}\right)$ (from (2.7) and (2.18)), $\left\|\phi_{x, \lambda}\right\|_{6}=\mathcal{O}\left((d \lambda)^{-1 / 2}\right.$ ) (from [24, Prop.1 (c)]) and $\left\|U_{x, \lambda}\right\|_{4}^{4}=\mathcal{O}\left(\lambda^{-1}\right)$, so the remainder term is $o\left((d \lambda)^{-1}\right)$.

Lemma A.2 As $\lambda \rightarrow \infty$, uniformly in $x \in \Omega$,

$$
\left\|P U_{x, \lambda}\right\|_{6 / 5}=\mathcal{O}\left(\lambda^{-1 / 2}\right) .
$$

Moreover, for $x$ in compact subset of $\Omega$,

$$
\begin{gathered}
\left\|\partial_{\lambda} P U_{x, \lambda}\right\|_{6 / 5}=\mathcal{O}\left(\lambda^{-3 / 2}\right), \\
\left\|\psi_{x, \lambda}-U_{x, \lambda}\right\|_{\infty}=\mathcal{O}\left(\lambda^{-1 / 2}\right)
\end{gathered}
$$

and

$$
\left\|\psi_{x, \lambda}\right\|_{6 / 5}=\mathcal{O}\left(\lambda^{-1 / 2}\right)
$$

Proof The bound (A.5) follows from $0 \leq P U_{x, \lambda} \leq U_{x, \lambda}$ (see [24, Prop.1(a)]) and a straightforward computation for $U_{x, \lambda}$, using the fact that $\Omega$ is bounded.

To prove (A.6) we first note that, by a straightforward computation, the claimed bound holds with $\partial_{\lambda} U_{x, \lambda}$ instead of $\partial_{\lambda} P U_{x, \lambda}$. The claimed bound now follows since by the bound on $\partial_{\lambda} U_{x, \lambda}-\partial_{\lambda} P U_{x, \lambda}$ in [24, Prop. 1 (c)] (which holds even in $L^{6}$ ).

For the proof of (A.7) we write $\psi_{x, \lambda}-U_{x, \lambda}=-\lambda^{-1 / 2} H_{a}(x, \cdot)-f_{x, \lambda}$. Then (A.7) follows from (2.6) and (2.18). Finally, (A.8) follows from (A.5) and (A.7). 


\section{A.2 Proof of (6.15)}

We have

$$
\begin{aligned}
N_{1}= & \beta^{2} \lambda^{-2} \int_{\Omega}\left|\nabla P U_{x, \lambda}\right|^{2} d y+\gamma^{2} \int_{\Omega}\left|\nabla \partial_{\lambda} P U_{x, \lambda}\right|^{2} d y \\
& +2 \beta \lambda^{-1} \int_{\Omega} \nabla P U_{x, \lambda} \cdot \nabla \partial_{\lambda} P U_{x, \lambda} d y \\
& +2 \beta \lambda^{-1} \int_{\Omega} \nabla \psi_{x, \lambda} \cdot \nabla P U_{x, \lambda} d y+2 \gamma \int_{\Omega} \nabla \psi_{x, \lambda} \cdot \nabla \partial_{\lambda} P U_{x, \lambda} d y \\
& +2 \beta \lambda^{-1} \int_{\Omega} a \psi_{x, \lambda} P U_{x, \lambda} d y+2 \gamma \int_{\Omega} a \psi_{x, \lambda} \partial_{\lambda} P U_{x, \lambda} d y .
\end{aligned}
$$

Therefore (6.15) will follow from the following relations, together with the facts that $\phi_{a}(x)=$ $o(1)$ by Proposition 5.1 and that $\beta, \gamma=\mathcal{O}(1)$ by Lemma 6.1,

$$
\begin{aligned}
\lambda^{-2} \int_{\Omega}\left|\nabla P U_{x, \lambda}\right|^{2} d y & =\frac{3 \pi^{2}}{4} \lambda^{-2}+o\left(\lambda^{-2}\right), \\
\int_{\Omega}\left|\nabla \partial_{\lambda} P U_{x, \lambda}\right|^{2} d y & =\frac{15 \pi^{2}}{64} \lambda^{-2}+o\left(\lambda^{-2}\right), \\
\lambda^{-1} \int_{\Omega} \nabla P U_{x, \lambda} \cdot \nabla \partial_{\lambda} P U_{x, \lambda} d y & =o\left(\lambda^{-2}\right), \\
\lambda^{-1} \int_{\Omega} \nabla \psi_{x, \lambda} \cdot \nabla P U_{x, \lambda} d y & =\frac{3 \pi^{2}}{4} \lambda^{-1}-4 \pi \phi_{a}(x) \lambda^{-2}+o\left(\lambda^{-2}\right), \\
\int_{\Omega} \nabla \psi_{x, \lambda} \cdot \nabla \partial_{\lambda} P U_{x, \lambda} d y & =2 \pi \phi_{a}(x) \lambda^{-2}+o\left(\lambda^{-2}\right), \\
\lambda \int_{\Omega} a \psi_{x, \lambda} P U_{x, \lambda} d y & =4 \pi\left(\phi_{a}(x)-\phi_{0}(x)\right) \lambda^{-2}+o\left(\lambda^{-2}\right), \\
\int_{\Omega} a \psi_{x, \lambda} \partial_{\lambda} P U_{x, \lambda} d y & =-2 \pi\left(\phi_{a}(x)-\phi_{0}(x)\right) \lambda^{-2}+o\left(\lambda^{-2}\right) .
\end{aligned}
$$

For the proof of these bounds we recall that $d \gtrsim 1$ by Proposition 4.1 .

The bounds (A.9), (A.10) and (A.11) follow from [24, (B.2), (B.7) and (B.5)], respectively.

For the proof of the remaining assertions we decompose $\psi_{x, \lambda}=U_{x, \lambda}-\lambda^{-1 / 2} H_{a}(x, \cdot)-$ $f_{x, \lambda}$ and recall the bound (2.18) on $f_{x, \lambda}$.

Proof of (A.12) By (1.9) and (2.15),

$$
\lambda^{-1} \int_{\Omega} \nabla \psi_{x, \lambda} \cdot \nabla P U_{x, \lambda} d y=3 \lambda^{-1} \int_{\Omega} U_{x, \lambda}^{5}\left(U_{x, \lambda}-\lambda^{-1 / 2} H_{a}(x, \cdot)\right) d y+o\left(\lambda^{-2}\right) .
$$

By (A.3), $3 \lambda^{-1} \int_{\Omega} U_{x, \lambda}^{6} d y=\frac{3 \pi^{2}}{4} \lambda^{-1}+o\left(\lambda^{-2}\right)$. On the other hand, by Lemma 2.5 ,

$$
3 \lambda^{-3 / 2} \int_{\Omega} U_{x, \lambda}^{5} H_{a}(x, y) d y=4 \pi \phi_{a}(x) \lambda^{-2}+o\left(\lambda^{-2}\right) .
$$

Proof of (A.13) By differentiating (1.9) and (2.15),

$$
\int_{\Omega} \nabla \psi_{x, \lambda} \cdot \nabla \partial_{\lambda} P U_{x, \lambda} d y=15 \int_{\Omega}\left(U_{x, \lambda}-\lambda^{-1 / 2} H_{a}(x, y)\right) U_{x, \lambda}^{4} \partial_{\lambda} U_{x, \lambda} d y+o\left(\lambda^{-2}\right) .
$$


To compute the first summand, we use $\int_{\mathbb{R}^{3}} U_{x, \lambda}^{5} \partial_{\lambda} U_{x, \lambda} d y=\partial_{\lambda} \int_{\mathbb{R}^{3}} U_{x, \lambda}^{6} d y=0$ and thus

$$
\begin{aligned}
& \left|\int_{\Omega} U_{x, \lambda}^{5} \partial_{\lambda} U_{x, \lambda} d y\right|=\left|\int_{\mathbb{R}^{3} \backslash \Omega} U_{x, \lambda}^{5} \partial_{\lambda} U_{x, \lambda} d y\right| \\
& \quad \leq(2 \lambda)^{-1} \int_{\mathbb{R}^{3} \backslash B_{\lambda d}(x)} \frac{|1-| x-\left.z\right|^{2} \mid}{\left(1+|x-z|^{2}\right)^{4}} d z=\mathcal{O}\left(\lambda^{-4}\right) .
\end{aligned}
$$

To compute the second summand we argue similarly as in the proof of Lemmas 2.5 and 2.6 and obtain

$$
-15 \lambda^{-1 / 2} \int_{\Omega} H_{a}(x, y) U_{x, \lambda}^{4} \partial_{\lambda} U_{x, \lambda} d y=2 \pi \phi_{a}(x) \lambda^{-2}+o\left(\lambda^{-2}\right) .
$$

The constant comes from

$$
\int_{\mathbb{R}^{3}} U_{x, \lambda}^{4} \partial_{\lambda} U_{x, \lambda} d y=2 \pi \lambda^{-3 / 2} \int_{0}^{\infty} \frac{\left(1-t^{2}\right) t^{2} d t}{\left(1+t^{2}\right)^{7 / 2}}=-\frac{2 \pi}{15} \lambda^{-3 / 2} .
$$

Proof of (A.14) Since $P U_{x, \lambda}=U_{x, \lambda}-\lambda^{-1 / 2} H_{0}(x, \cdot)-f_{x, \lambda}$,

$$
\lambda^{-1} \int_{\Omega} a \psi_{x, \lambda} P U_{x, \lambda} d y=\lambda^{-1} \int_{\Omega} a\left(U_{x, \lambda}-\lambda^{-1 / 2} H_{a}(x, y)\right)\left(U_{x, \lambda}-\lambda^{-1 / 2} H_{0}(x, y)\right) d y+o\left(\lambda^{-2}\right) .
$$

We have

$\lambda^{-1} \int_{\Omega} a U_{x, \lambda}^{2} d y=\lambda^{-2} \int_{\Omega} a(y) \frac{1}{\lambda^{-2}+|x-y|^{2}} d y=\lambda^{-2} \int_{\Omega} a(y) \frac{1}{|x-y|^{2}} d y+o\left(\lambda^{-2}\right)$

and, similarly,

$$
-\lambda^{-3 / 2} \int_{\Omega} a U_{x, \lambda}\left(H_{a}(x, y)+H_{0}(x, y)\right) d y=-\lambda^{-2} \int_{\Omega} a(y) \frac{H_{a}(x, y)+H_{0}(x, y)}{|x-y|} d y+o\left(\lambda^{-2}\right) .
$$

Putting everything together and recalling that $G_{a}(x, y)=\frac{1}{|x-y|}-H_{a}(x, y)$, we obtain

$$
\begin{aligned}
\lambda^{-1} \int_{\Omega} a \psi_{x, \lambda} P U_{x, \lambda} d y & =\lambda^{-2} \int_{\Omega} a(y) G_{a}(x, y) G_{0}(x, y) d y+o\left(\lambda^{-2}\right) \\
& =4 \pi\left(\phi_{a}(x)-\phi_{0}(x)\right) \lambda^{-2}+o\left(\lambda^{-2}\right),
\end{aligned}
$$

where the last equality follows from the resolvent identity (2.8).

Proof of (A.15) Since $\left\|\partial_{\lambda} f_{x, \lambda}\right\|_{\infty}=\mathcal{O}\left(\lambda^{-7 / 2}\right)$ by [24, Prop. 1 (c)], we get, similarly as before,

$$
\int_{\Omega} a \psi_{x, \lambda} \partial_{\lambda} P U_{x, \lambda} d y=\int_{\Omega} a\left(U_{x, \lambda}-\lambda^{-1 / 2} H_{a}(x, y)\right)\left(\partial_{\lambda} U_{x, \lambda}+\frac{1}{2} \lambda^{-3 / 2} H_{0}(x, y)\right) d y+o\left(\lambda^{-2}\right) .
$$

We have

$\int_{\Omega} a U_{x, \lambda} \partial_{\lambda} U_{x, \lambda} d y=\frac{1}{2} \lambda^{-2} \int_{\Omega} a(y) \frac{\lambda^{-2}-|x-y|^{2}}{\left(\lambda^{-2}+|x-y|^{2}\right)^{2}} d y=-\frac{1}{2} \lambda^{-2} \int_{\Omega} a(y) \frac{1}{|x-y|^{2}} d y+o\left(\lambda^{-2}\right)$

and, similarly,

$$
-\lambda^{-1 / 2} \int_{\Omega} a H_{a}(x, y) \partial_{\lambda} U_{x, \lambda} d y=\frac{1}{2} \lambda^{-2} \int_{\Omega} a(y) \frac{H_{a}(x, y)}{|x-y|} d y
$$

and

$$
\lambda^{-3 / 2} \int_{\Omega} a U_{x, \lambda} H_{0}(x, y) d y=\lambda^{-2} \int_{\Omega} a(y) \frac{H_{0}(x, y)}{|x-y|} d y+o\left(\lambda^{-2}\right) .
$$


Putting everything together and using the resolvent identity (2.8) as in the proof of (A.14), we obtain (A.15).

This completes the proof of (6.15).

\section{A.3 Proof of (6.16)}

We have

$$
\begin{aligned}
D_{1}= & 6 \beta \lambda^{-1} \int_{\Omega} \psi_{x, \lambda}^{5} P U_{x, \lambda} d y+6 \gamma \int_{\Omega} \psi_{x, \lambda}^{5} \partial_{\lambda} P U_{x, \lambda} d y \\
& +15 \beta^{2} \lambda^{-2} \int_{\Omega} \psi_{x, \lambda}^{4} P U_{x, \lambda}^{2} d y+15 \gamma^{2} \int_{\Omega} \psi_{x, \lambda}^{4}\left(\partial_{\lambda} P U_{x, \lambda}\right)^{2} d y+30 \beta \gamma \lambda^{-1} \\
& \int_{\Omega} \psi_{x, \lambda}^{4} P U_{x, \lambda} \partial_{\lambda} P U_{x, \lambda} d y .
\end{aligned}
$$

Therefore (6.16) will follow from the following relations, together with the facts that $\phi_{a}(x)=$ $o(1)$ by Proposition 5.1 and that $\beta, \gamma=\mathcal{O}(1)$ by Lemma 6.1,

$$
\begin{aligned}
\lambda^{-1} \int_{\Omega} \psi_{x, \lambda}^{5} P U_{x, \lambda} d y & =\frac{\pi^{2}}{4} \lambda^{-1}-\frac{4 \pi}{3}\left(5 \phi_{a}(x)+\phi_{0}(x)\right) \lambda^{-2}+o\left(\lambda^{-2}\right), \\
\int_{\Omega} \psi_{x, \lambda}^{5} \partial_{\lambda} P U_{x, \lambda} d y & =\frac{2 \pi}{3}\left(\phi_{a}(x)+\phi_{0}(x)\right) \lambda^{-2}+o\left(\lambda^{-2}\right) \\
\lambda^{-2} \int_{\Omega} \psi_{x, \lambda}^{4} P U_{x, \lambda}^{2} d y & =\frac{\pi^{2}}{4} \lambda^{-2}+o\left(\lambda^{-2}\right) \\
\int_{\Omega} \psi_{x, \lambda}^{4}\left(\partial_{\lambda} P U_{x, \lambda}\right)^{2} d y & =\frac{\pi^{2}}{64} \lambda^{-2}+o\left(\lambda^{-2}\right), \\
\lambda^{-1} \int_{\Omega} \psi_{x, \lambda}^{4} P U_{x, \lambda} \partial_{\lambda} P U_{x, \lambda} d y & =o\left(\lambda^{-2}\right) .
\end{aligned}
$$

Proof of (A.16) We insert $\psi_{x, \lambda}=U_{x, \lambda}-\lambda^{-1 / 2} H_{a}(x, \cdot)-f_{x, \lambda}$ and $P U_{x, \lambda}=U_{x, \lambda}-$ $\lambda^{-1 / 2} H_{0}(x, \cdot)-f_{x, \lambda}$ to obtain

$$
\lambda^{-1} \int_{\Omega} \psi_{x, \lambda}^{5} P U_{x, \lambda} d y=\lambda^{-1} \int_{\Omega} U_{x, \lambda}^{6} d y-\lambda^{-3 / 2} \int_{\Omega} U_{x, \lambda}^{5}\left(5 H_{a}(x, y)+H_{0}(x, y)\right) d y+o\left(\lambda^{-2}\right) .
$$

For the first term we use (A.3) and for the second term we use Lemma 2.5.

Proof of (A.17) Similarly as before, we obtain

$$
\begin{aligned}
\int_{\Omega} \psi_{x, \lambda}^{5} \partial_{\lambda} P U_{x, \lambda} d y= & \int_{\Omega} U_{x, \lambda}^{5} \partial_{\lambda} U_{x, \lambda} d y-5 \lambda^{-1 / 2} \int_{\Omega} U_{x, \lambda}^{4} \partial_{\lambda} U_{x, \lambda} H_{a}(x, y) d y \\
& +\frac{1}{2} \lambda^{-3 / 2} \int_{\Omega} U_{x, \lambda}^{5} H_{0}(x, y) d y+o\left(\lambda^{-2}\right) .
\end{aligned}
$$

For the first and the second term we argue as in the proof of (A.13) and for the third one we use Lemma 2.5.

The bounds (A.18), (A.19) and (A.20) follow from the corresponding relations where $\psi_{x, \lambda}$ and $P U_{x, \lambda}$ are replaced by $U_{x, \lambda}$ and where $\partial_{\lambda} P U_{x, \lambda}$ is replaced by $\partial_{\lambda} U_{x, \lambda}$.

This completes the proof of (6.16). 


\section{Appendix B : Proof of Proposition 3.1}

In this appendix we provide a proof of the approximate form of almost minimizers. This result is probably well-known to specialists.

Proof of Proposition 3.1 Step 1 We show that $u_{\epsilon} \rightarrow 0$ in $H_{0}^{1}(\Omega)$.

The assumptions imply that $\left(u_{\epsilon}\right)$ is bounded in $H_{0}^{1}(\Omega)$ and therefore it has a weak limit point. Let $u_{0} \in H_{0}^{1}(\Omega)$ be such a limit point and write $r_{\epsilon}:=u_{\epsilon}-u_{0}$. In the remainder of this step we restrict ourselves to values of $\epsilon$ along which $r_{\epsilon} \rightarrow 0$ in $H_{0}^{1}(\Omega)$. By Rellich's compactness theorem $r_{\epsilon} \rightarrow 0$ in $L^{2}(\Omega)$ and, passing to a subsequence if necessary, we may assume that $r_{\epsilon} \rightarrow 0$ almost everywhere in $\Omega$. By weak convergence in $H_{0}^{1}(\Omega)$ and strong convergence in $L^{2}(\Omega)$ we have

$$
\begin{aligned}
3^{-1 / 2} S^{3 / 2}+o(1) & =\int_{\Omega}\left(\left|\nabla u_{\epsilon}\right|^{2}+a u_{\epsilon}^{2}+\epsilon V u_{\epsilon}^{2}\right) d x \\
& =\int_{\Omega}\left(\left|\nabla u_{0}\right|^{2}+a u_{0}^{2}\right) d x+\int_{\Omega}\left|\nabla r_{\epsilon}\right|^{2} d x+o(1) .
\end{aligned}
$$

Thus,

$$
T:=\lim _{\epsilon \rightarrow 0} \int_{\Omega}\left|\nabla r_{\epsilon}\right|^{2} d x \text { exists and satisfies } \quad 3^{-1 / 2} S^{3 / 2}=\int_{\Omega}\left(\left|\nabla u_{0}\right|^{2}+a u_{0}^{2}\right) d x+T .
$$

On the other hand, by the almost everywhere convergence and the Brézis-Lieb lemma [7],

$$
(S / 3)^{3 / 2}=\int_{\Omega} u_{\epsilon}^{6} d x=\int_{\Omega} u_{0}^{6} d x+\int_{\Omega} r_{\epsilon}^{6} d x+o(1) .
$$

Thus,

$$
M:=\lim _{\epsilon \rightarrow 0} \int_{\Omega} r_{\epsilon}^{6} d x \quad \text { exists and satisfies } \quad(S / 3)^{3 / 2}=\int_{\Omega} u_{0}^{6} d x+M .
$$

We conclude that

$$
S==\lim _{\epsilon \rightarrow 0} \mathcal{S}_{a+\epsilon V}\left[u_{\epsilon}\right]=\frac{\int_{\Omega}\left(\left|\nabla u_{0}\right|^{2}+a u_{0}^{2}\right) d x+T}{\left(\int_{\Omega} u_{0}^{6} d x+M\right)^{1 / 3}} .
$$

In the denominator, we bound

$$
\left(\int_{\Omega} u_{0}^{6} d x+M\right)^{1 / 3} \leq\left(\int_{\Omega} u_{0}^{6} d x\right)^{1 / 3}+M^{1 / 3}
$$

and in the numerator we bound $T \geq S M^{1 / 3}$. Rearranging terms, we thus obtain

$$
S\left(\int_{\Omega} u_{0}^{6} d x\right)^{1 / 3} \geq \int_{\Omega}\left(\left|\nabla u_{0}\right|^{2}+a u_{0}^{2}\right) d x .
$$

Since the opposite inequality holds as well by definition of $S(a)$ and the assumption that $S(a)=S$, we need to have, in particular, equality in (B.1). It is elementary to see that this holds if and only if either $\int u_{0}^{6} d x=0$ (that is, $u_{0} \equiv 0$ ) or if $M=0$.

Let us rule out the case $M=0$. If we had $M=0$, then, in particular, $u_{0} \not \equiv 0$ and therefore $u_{0}$ would be a minimizer for the $S(a)$ problem. However, as shown by Druet (Step 1 in [12]), the $S(a)$ problem does not have a minimizer. (Note that this part of Druet's paper does not need any regularity of $a$.) Thus, $M>0$, which, as explained before, implies $u_{0} \equiv 0$. 
Step 2 We show that along a subsequence,

$$
u_{\epsilon}=s U_{z_{\epsilon}, \mu_{\epsilon}}+\sigma_{\epsilon}
$$

with $s \in\{ \pm 1\}, z_{\epsilon} \rightarrow x_{0} \in \bar{\Omega}, \mu_{\epsilon} \operatorname{dist}\left(z_{\epsilon}, \partial \Omega\right) \rightarrow \infty$ and $\sigma_{\epsilon} \rightarrow 0$ in $\dot{H}^{1}\left(\mathbb{R}^{3}\right)$.

Indeed, by Step 1 and Rellich's compactness theorem we have $u_{\epsilon} \rightarrow 0$ in $L^{2}(\Omega)$ and therefore

$$
\frac{\int_{\Omega}\left|\nabla u_{\epsilon}\right|^{2} d x}{\left(\int_{\Omega} u_{\epsilon}^{6} d x\right)^{1 / 3}} \rightarrow S
$$

Thus, the $u_{\epsilon}$, extended by zero to functions in $\dot{H}^{1}\left(\mathbb{R}^{3}\right)$, form a minimizing sequence for the Sobolev quotient. By a theorem of Lions [22] there exist $\left(z_{\epsilon}\right) \subset \mathbb{R}^{3}$ and $\left(\mu_{\epsilon}\right) \subset \mathbb{R}_{+}$ such that, along a subsequence, $\mu_{\epsilon}^{-1 / 2} u_{\epsilon}\left(\mu_{\epsilon}^{-1} \cdot+z_{\epsilon}\right)$ converges in $\dot{H}^{1}\left(\mathbb{R}^{3}\right)$ to a function that is an optimizer for the Sobolev inequality. By the classification of these optimizers (which appears, for instance, in [22, Cor. I.1]) and taking the normalization of the $u_{\epsilon}$ into account, we can assume, after modifying the $\mu_{\epsilon}$ and $z_{\epsilon}$, that

$$
\mu_{\epsilon}^{-1 / 2} u_{\epsilon}\left(\mu_{\epsilon}^{-1} \cdot+z_{\epsilon}\right) \rightarrow s U_{0,1} \text { in } \dot{H}^{1}\left(\mathbb{R}^{3}\right)
$$

for some $s \in\{ \pm 1\}$. By a change of variables (which preserves the $\dot{H}^{1}\left(\mathbb{R}^{3}\right)$ norm) this is the same as (B.2).

Note that

$$
\int_{\mathbb{R}^{3}} U^{6} d x=\int_{\Omega} u_{\epsilon}^{6} d x=\int_{\Omega}\left(s U_{z_{\epsilon}, \mu_{\epsilon}}+\sigma_{\epsilon}\right)^{6} d x=\int_{\Omega} U_{z_{\epsilon}, \mu_{\epsilon}}^{6} d x+o(1) .
$$

Thus, $\mu_{\epsilon} \rightarrow \infty$ and $\operatorname{dist}\left(z_{\epsilon}, \Omega\right) \rightarrow 0$. Using, in addition, the fact that the boundary of $\Omega$ is $C^{1}$, we conclude that $\mu_{\epsilon} \operatorname{dist}\left(z_{\epsilon}, \mathbb{R}^{3} \backslash \Omega\right) \rightarrow \infty$. In particular, after passing to a subsequence, $z_{\epsilon} \rightarrow x_{0} \in \bar{\Omega}$.

Step 3 We now conclude the proof of the proposition.

Since the remaining arguments are similar to those in [24, Prop. 2] we omit most of the details. As in that paper, the conclusions from Step 2 allow us to apply the result of Bahri-Coron [5, Prop. 7] and lead to a decomposition

$$
u_{\epsilon}=\alpha_{\epsilon} P U_{x_{\epsilon}, \lambda_{\epsilon}}+w_{\varepsilon}
$$

with $x_{\epsilon} \in \Omega$, bounded $\alpha_{\epsilon}$ and $w_{\epsilon} \in T_{x_{\epsilon}, \lambda_{\epsilon}}^{\perp}$ such that $w_{\epsilon} \rightarrow 0$ in $H_{0}^{1}(\Omega)$. This implies

$$
\int_{\Omega}\left|\nabla\left(\alpha_{\epsilon} P U_{x_{\epsilon}, \lambda_{\epsilon}}\right)\right|^{2} d y=\int_{\Omega}\left|\nabla u_{\epsilon}\right|^{2} d y+o(1)=3^{-1 / 2} S^{3 / 2}+o(1) .
$$

By the same argument as in [24, Prop. 2] with $3^{-1 / 2} S^{3 / 2}$ instead of $\mu$ on the right side of [24, (2.18)] we infer that $\lambda_{\epsilon} / \mu_{\epsilon}+\mu_{\epsilon} / \lambda_{\epsilon}+\lambda_{\epsilon} \mu_{\epsilon}\left|x_{\epsilon}-z_{\epsilon}\right| \lesssim 1$. From this we conclude that $\lambda_{\epsilon} \rightarrow \infty, x_{\epsilon} \rightarrow x_{0}$ and $\lambda_{\epsilon} \operatorname{dist}\left(x_{\varepsilon}, \partial \Omega\right) \rightarrow \infty$. Finally, using [24, (B.2)], $\alpha_{\epsilon} \rightarrow s$. The last relation allows us to replace $w_{\epsilon}$ by $\alpha_{\epsilon} w_{\epsilon}$, which still has the same properties, and obtain the decomposition stated in the proposition. This completes the proof.

\section{References}

1. Amar, M., Garroni, A.: Г-convergence of concentration problems. Ann. Sc. Norm. Super. Pisa Cl. Sci. (5) 2(1), 151-179 (2003)

2. Atkinson, F.V., Peletier, L.A.: Elliptic equations with nearly critical growth. J. Differ. Equ. 70(3), 349-365 (1987) 
3. Aubin, T.: Problèmes isoperimétriques et espaces de Sobolev. J. Differ. Geom. 11, 573-598 (1976)

4. Bahri, A.: Critical Points at Infinity in Some Variational Problems. Pitman Research Notes in Mathematics Series, vol. 182. Longman Scientific \& Technical, Harlow (1989)

5. Bahri, A., Coron, J.-M.: On a nonlinear elliptic equation involving the critical Sobolev exponent: the effect of the topology of the domain. Commun. Pure Appl. Math. 41(3), 253-294 (1988)

6. Brézis, H.: Elliptic equations with limiting Sobolev exponents-the impact of topology. Frontiers of the mathematical sciences: 1985 (New York, 1985). Commun. Pure Appl. Math. 39(S suppl), S17-S39 (1986)

7. Brézis, H., Lieb, E.H.: A relation between pointwise convergence of functions and convergence of functionals. Proc. Am. Math. Soc. 88(3), 486-490 (1983)

8. Brézis, H., Nirenberg, L.: Positive solutions of nonlinear elliptic equations involving critical Sobolev exponents. Commun. Pure Appl. Math. 36, 437-477 (1983)

9. Brézis, H., Peletier, L.A.: Asymptotics for elliptic equations involving critical growth. In: Partial Differential Equations and the Calculus of Variations, vol. I, pp. 149-192. Progress in Nonlinear Differential Equations Applications, vol. 1. Birkhäuser Boston, Boston, MA (1989)

10. Budd, C.: Semilinear elliptic equations with near critical growth rates. Proc. R. Soc. Edinb. Sect. A 107(3-4), 249-270 (1987)

11. Davies, E.B.: Heat Kernels and Spectral Theory. Cambridge University Press, Cambridge (1989)

12. Druet, O.: Elliptic equations with critical Sobolev exponents in dimension 3. Ann. Inst. H. Poincaré-AN 19, 125-142 (2002)

13. Druet, O., Hebey, E., Robert, F.: Blow-up Theory for Elliptic PDEs in Riemannian Geometry. Mathematical Notes, vol. 45. Princeton University Press, Princeton (2004)

14. Ekholm, T., Frank, R.L., Kovařík, H.: Weak perturbations of the $p$-Laplacian. Calc. Var. Partial Differ. Equ. 53(3-4), 781-801 (2015)

15. Esposito, P.: On some conjectures proposed by Haim Brezis. Nonlinear Anal. 54, 751-759 (2004)

16. Flucher, M.: Variational problems with concentration. In: Progress in Nonlinear Differential Equations and their Applications, vol. 36. Birkhäuser Verlag, Basel (1999)

17. Flucher, M., Garroni, A., Müller, S.: Concentration of low energy extremals: identification of concentration points. Calc. Var. Partial Differ. Equ. 14(4), 483-516 (2002)

18. Frank, R.L., König, T., Kovařík, H.: Energy asymptotics in the Brezis-Nirenberg problem. The higherdimensional case. Math. Eng. 2(1), 119-140 (2020)

19. Han, Z.-C.: Asymptotic approach to singular solutions for nonlinear elliptic equations involving critical Sobolev exponent. Ann. Inst. H. Poincaré Anal. Non Linéaire 8(2), 159-174 (1991)

20. Hebey, E., Vaugon, M.: From best constants to critical functions. Math. Z. 237(4), 737-767 (2001)

21. Lieb, E.H., Loss, M.: Analysis, 2nd edn. In: Graduate Studies in Mathematics, vol. 14. American Mathematical Society, Providence (2001)

22. Lions, P.-L.: The concentration-compactness principle in the calculus of variations. The limit case. I. Rev. Mat. Iberoamericana 1(1), 145-201 (1985)

23. Rey, O.: Proof of two conjectures of H. Brezis and L.A. Peletier. Manuscr. Math. 65, 19-37 (1989)

24. Rey, O.: The role of the Green's function in a non-linear elliptic equation involving the critical Sobolev exponent. J. Funct. Anal. 89, 1-52 (1990)

25. Rodemich, E.: The Sobolev inequality with best possible constant. In: Analysis Seminar Caltech, Spring (1966)

26. Rosen, G.: Minimum value for $c$ in the Sobolev inequality $\left\|\phi^{3}\right\| \leq c\|\nabla \phi\|^{3}$. SIAM J. Appl. Math. 21, 30-32 (1971)

27. Schoen, R.: Conformal deformation of a Riemannian metric to constant scalar curvature. J. Differ. Geom. 20(2), 479-495 (1984)

28. Simon, B.: The bound state of weakly coupled Schrödinger operators in one and two dimensions. Ann. Phys. 97, 279-288 (1976)

29. Struwe, M.: A global compactness result for elliptic boundary value problems involving limiting nonlinearities. Math. Z. 187(4), 511-517 (1984)

30. Takahashi, F.: On the location of blow up points of least energy solutions to the Brezis-Nirenberg equation. Funkcial. Ekvac. 47(1), 145-166 (2004)

31. Talenti, G.: Best constants in Sobolev inequality. Ann. Mat. Pura Appl. 110, 353-372 (1976)

32. Wei, J.: Asymptotic behavior of least energy solutions to a semilinear Dirichlet problem near the critical exponent. J. Math. Soc. Jpn. 50(1), 139-153 (1998)

Publisher's Note Springer Nature remains neutral with regard to jurisdictional claims in published maps and institutional affiliations. 